This is a self-archived version of an original article. This version may differ from the original in pagination and typographic details.

Author(s): Manninen, Juuso; Asjad, Muhammad; Ojajärvi, Risto; Kuusela, Petri; Massel,

Title: Clauser-Horne-Shimony-Holt Bell inequality test in an optomechanical device

Year: 2018

Version: Published version

Copyright: @ 2018 American Physical Society

Rights: In Copyright

Rights url: http://rightsstatements.org/page/InC/1.0/?language=en

Please cite the original version:

Manninen, J., Asjad, M., Ojajärvi, R., Kuusela, P., \& Massel, F. (2018). Clauser-Horne-ShimonyHolt Bell inequality test in an optomechanical device. Physical Review A, 98(4), Article 043831. https://doi.org/10.1103/physreva.98.043831 


\title{
Clauser-Horne-Shimony-Holt Bell inequality test in an optomechanical device
}

\author{
Juuso Manninen, ${ }^{1}$ Muhammad Asjad, ${ }^{2}$ Risto Ojajärvi, ${ }^{2}$ Petri Kuusela, ${ }^{2}$ and Francesco Massel $^{2, *}$ \\ ${ }^{1}$ Department of Applied Physics, Low Temperature Laboratory, Aalto University, P.O. Box 15100, FI-00076 AALTO, Finland \\ ${ }^{2}$ Department of Physics and Nanoscience Center, University of Jyväskylä, P.O. Box 35 (YFL), FI-40014 University of Jyväskylä, Finland
}

(Received 27 February 2018; revised manuscript received 21 August 2018; published 15 October 2018)

\begin{abstract}
We propose here a scheme based on the measurement of quadrature phase coherence that is aimed at testing the Clauser-Horne-Shimony-Holt Bell inequality in an optomechanical setting. Our setup is constituted by two optical cavities dispersively coupled to a common mechanical resonator. We show that it is possible to generate Einstein-Podolsky-Rosen-like correlations between the quadratures of the output fields of the two cavities and, depending on the system parameters, to observe the violation of the Clauser-Horne-Shimony-Holt inequality.
\end{abstract}

DOI: 10.1103/PhysRevA.98.043831

\section{INTRODUCTION}

In his seminal work, motivated by the work by Einstein, Podolsky, and Rosen [1], Bell showed that theories relying on local (possibly hidden) variables, which are bound to satisfy certain inequalities, cannot describe all quantum-mechanical predictions [2]. From the point of view of quantum theory, a violation of these Bell inequalities (BIs) necessarily implies entanglement between spatially separated subsystems [3]. Beyond their intrinsic conceptual relevance, BI tests have potentially important technological repercussions, allowing one to certify the security of quantum cryptographic schemes [4], making it relevant to explore the possibility of performing such tests in different setups and for different physical systems.

Since the work of Bell, multiple experimental realizations of BI tests have been conducted [5-18], the first one being performed by Freedman and Clauser [5]. However, the confirmation that, without any additional assumptions, i.e., closing all loopholes, predictions offered by locally realistic theories cannot reproduce the experimental results has been obtained only in the last few years [15-17]. Even more recently, based on an early theoretical proposal [19] and resorting to an experimental setup similar to that employed in the Bell test performed by $\mathrm{Ou}$ and Mandel [20], a BI test relying on continuous variable measurement has been performed [18].

Owing to the recent progresses in the concomitant manipulation of mechanical and optical degrees of freedom at the quantum level [21,22], cavity optomechanical systems represent one of the cornerstones for future quantum information and communication technologies. On a more fundamental level, these systems represent one of the most promising platforms for experimental verification of physical theories, with applications ranging from gravitational wave detection [23] to the potential observation of quantum gravitational effects [24] and entanglement between nearly macroscopic mechanical objects [25-29].

\footnotetext{
*francesco.p.massel@jyu.fi
}

In this spirit, in this article, we investigate the test of the Clauser-Horne-Shimony-Holt (CHSH) [30] BI in an optomechanical system. Our main focus is a two-cavity optomechanical setup, either in the microwave or in the visible-light regime, allowing for unrivaled flexibility in the choice of detectors and transmission lines for loophole-free tests. In addition, the nature of the optomechanical interaction characterizing our proposal opens up the possibility for BI tests in mixed microwave and optical settings [31]. The two-cavitiesone-mechanics setup, which we consider here for the BI test was discussed in the past in connection with the entanglement properties of optomechanical systems [25,31,32] and was experimentally realized in the context of multimode quantum signal amplification of microwaves [33]. While other ideas for testing BIs in an optomechanical setting have recently been proposed [34,35], they are based on a rather different setup than the one discussed here, for which, due to the sequential nature of the pulsing scheme, closing all loopholes, in particular the locality loophole, requires addressing extra technical difficulties, as discussed in the supplementary material of Ref. [34] which are not present in the setup discussed here. On more general grounds, it is worth mentioning that closing the locality loophole in a microwave setting represents a formidable challenge due to the necessity of the noiseless distribution of microwave signals. In this sense an all-optical realization of our proposal would thus seem favorable. In the following, however, in order to underline the relation to the present state-of-the-art experimental capabilities, we mainly focus on the experimental parameters of the microwave setup discussed in Ref. [33].

While the previous BI tests mentioned above rely either on the polarization degree of freedom of optical photons [5-9,13,14,16,17], or on different realizations of two-level systems in a condensed-matter context $[10-12,15]$, our proposal follows the ideas suggested by Tan et al. [36,37] and considers the possibility of a CHSH BI violation through the detection of the quadrature phases; in our case, in an optomechanical setting.

The paper is organized as follows: In Sec. II we introduce the model and discuss the conditions for the violation of the CHSH BI. In Sec. III we describe the numerical results for the 
violation of the BI and we show its sensitivity to variations of other system parameters. Lastly, we discuss the effect of various noise sources on the violation of the inequality in our setup.

\section{MODEL AND EQUATION OF MOTION}

The setup considered here is constituted by two electromagnetic resonant cavities ( $\mathrm{A}$ and $\mathrm{C}$ ) - either in the optical or microwave regime-dispersively coupled to a mechanical resonator. Following the standard description of optomechanical systems [22,38-41], the Hamiltonian for the system can be written as

$$
\begin{aligned}
H= & \omega_{\mathrm{a}} a^{\dagger} a+\omega_{\mathrm{c}} c^{\dagger} c+\omega_{\mathrm{m}} b^{\dagger} b \\
& +\left(g_{\mathrm{a}} a^{\dagger} a+g_{\mathrm{c}} c^{\dagger} c\right)\left(b^{\dagger}+b\right),
\end{aligned}
$$

where $\mathrm{a}, \mathrm{c}$, and $\mathrm{b}$ represent the lowering operators associated with cavity $\mathrm{A}$ and $\mathrm{C}$ and the mechanical modes, respectively; $\omega_{\mathrm{a}}, \omega_{\mathrm{c}}, \omega_{\mathrm{m}}$ are their resonant frequencies and $g_{\mathrm{a}}$ and $g_{\mathrm{c}}$ are the single-photon radiation-pressure couplings for modes a and c with the mechanical mode.

Along the lines of the experiment discussed in Ref. [33], we assume that each cavity is driven by a strong coherent tone $\alpha_{\mathrm{in}, \mathrm{a}}$ and $\alpha_{\mathrm{in, \textrm {c }}}$ (for cavity $\mathrm{A}$ and $\mathrm{C}$, respectively). We consider that the driving of each cavity is detuned from the cavity resonance: we assume cavity $\mathrm{A}$ to be driven with a frequency $\omega_{\mathrm{d}, \mathrm{a}}=\omega_{\mathrm{a}}+\omega_{\mathrm{m}}$ (blue mechanical sideband) and cavity $\mathrm{C}$ with a frequency $\omega_{\mathrm{d}, \mathrm{c}}=\omega_{\mathrm{c}}-\omega_{\mathrm{m}}$ (red mechanical sideband). In our analysis, we employ the usual description of the system in terms of quantum Langevin equations [42] for the fluctuations around the cavity fields induced by the drives. In this scenario, we consider the linearized dynamics of the fluctuations around the pump tones and replace $a \rightarrow a+\alpha_{\mathrm{A}}$ and $c \rightarrow c+\alpha_{\mathrm{C}}$ (see Appendix A).

Moving to a frame rotating at $\omega_{\mathrm{d}, \mathrm{a}}$ and $\omega_{\mathrm{d}, \mathrm{c}}$ for modes $a$ and $c$ respectively and, defining $\Delta_{\mathrm{x}}=\omega_{\mathrm{d}, \mathrm{x}}-\omega_{\mathrm{x}}(x=a, c)$, we obtain the following equations of motion for the fluctuations:

$$
\begin{aligned}
& \dot{a}=\left(-i \Delta_{\mathrm{a}}-\frac{\kappa_{\mathrm{a}}}{2}\right) a-i G_{+}\left(b^{\dagger}+b\right)+\sqrt{\kappa_{\mathrm{e}, \mathrm{a}}} a_{\mathrm{i}}+\sqrt{\kappa_{\mathrm{i}, \mathrm{a}}} a_{\mathrm{I}}, \\
& \dot{c}=\left(-i \Delta_{\mathrm{c}}-\frac{\kappa_{\mathrm{c}}}{2}\right) c-i G_{-}\left(b^{\dagger}+b\right)+\sqrt{\kappa_{\mathrm{e}, \mathrm{c}}} c_{\mathrm{i}}+\sqrt{\kappa_{\mathrm{i}, \mathrm{c}}} c_{\mathrm{I}}
\end{aligned}
$$

$\dot{b}=\left(-\omega_{\mathrm{m}}-\frac{\gamma}{2}\right) b-i G_{+}\left(a^{\dagger}+a\right)-i G_{-}\left(c^{\dagger}+c\right)+\sqrt{\gamma} b_{\mathrm{i}}$,

where $G_{+}=g_{a} \alpha_{\mathrm{A}}$ and $G_{-}=g_{c} \alpha_{\mathrm{C}}$ are the linearized optomechanical couplings, and $\kappa_{\mathrm{a}}, \kappa_{\mathrm{c}}$, and $\gamma$ are the linewidths of cavities $\mathrm{A}, \mathrm{C}$, and the mechanical resonator. Moreover, we have defined $a_{\mathrm{i}}, a_{\mathrm{I}}, c_{\mathrm{i}}, c_{\mathrm{I}}, b_{\mathrm{i}}$ to be the input operators associated with the external input and internal fields, respectively ( $i$ and $I$ ), for cavities A and $\mathrm{C}$ and the mechanics, respectively.

It is possible to obtain the expression of the cavity fields in frequency space by Fourier transforming Eqs. (2a)-(2c). The transformation leads to the following set of linear algebraic equations:

$$
\begin{aligned}
-i \omega a= & \left(-i \Delta_{\mathrm{a}}-\frac{\kappa_{\mathrm{a}}}{2}\right) a-i G_{+}\left(b^{\dagger}+b\right) \\
& +\sqrt{\kappa_{\mathrm{e}, \mathrm{a}}} a_{\mathrm{i}}+\sqrt{\kappa_{\mathrm{i}, \mathrm{a}}} a_{\mathrm{I}}, \\
-i \omega c= & \left(-i \Delta_{\mathrm{c}}-\frac{\kappa_{\mathrm{c}}}{2}\right) c-i G_{-}\left(b^{\dagger}+b\right) \\
& +\sqrt{\kappa_{\mathrm{e}, \mathrm{c}}} c_{\mathrm{i}}+\sqrt{\kappa_{\mathrm{i}, \mathrm{c}}} c_{\mathrm{I}}, \\
-i \omega b= & -\frac{\gamma}{2} b-i G_{+}\left(a^{\dagger}+a\right) \\
& -i G_{-}\left(c^{\dagger}+c\right)+\sqrt{\gamma} b_{\mathrm{i}},
\end{aligned}
$$

which can be solved through standard techniques. Furthermore, according to the input-output theory [42], the operators for the output fields of cavity A are related to the cavity operators and to the input noise operators by the relation $a_{\mathrm{o}}=\sqrt{\kappa_{\mathrm{e}, \mathrm{a}}} a-a_{\mathrm{i}}$, where $\kappa_{\mathrm{e}, \mathrm{a}}$ is the external coupling rate for cavity A-and analogously for cavity $\mathrm{C}$.

These relations, combined with the solution of Eqs. (3a)(3c), allow us to map the input cavity modes to the output fields $a_{\mathrm{o}}, c_{\mathrm{o}}$ in the frequency domain as

$$
\begin{aligned}
& a_{\mathrm{o}}=A_{\mathrm{d}} a_{\mathrm{i}}+A_{\mathrm{x}} c_{\mathrm{i}}^{\dagger}+\mathcal{N}_{\mathrm{a}}, \\
& c_{\mathrm{o}}=C_{\mathrm{d}} c_{\mathrm{i}}+C_{\mathrm{x}} a_{\mathrm{i}}^{\dagger}+\mathcal{N}_{\mathrm{c}},
\end{aligned}
$$

where the operators $\mathcal{N}_{\mathrm{a}}\left(\mathcal{N}_{\mathrm{c}}\right)$ account for the noise associated with the mechanical resonator and the internal losses of the cavity. In addition to these noise sources, we consider that the external ports of the device represent potential further noise sources (see Appendix B). While the direct solution of Eqs. (3a)-(3c) outlined above is sufficient to determine the value of the coefficients in Eq. (4a), a deeper physical intuition into the mechanism leading to the quantum correlations among the modes-required for the violation of the $\mathrm{BI}$ - can be obtained by resorting to the rotating-wave approximation (RWA): the full derivation of the expressions for the coefficients given in Eq. (4a) within the RWA is given in Appendix B, where we also compare RWA results with the full solution of Eqs. (3a)-(3c), which shows that, as it is usually the case, RWA and full results coincide in the so-called sideband resolved regime $\left(\omega_{\mathrm{m}} / \kappa \gg 1\right)$. We outline here the key points of such derivation. To do this, we write the equations of motion (EOMs) in a frame rotating at the resonant frequency of each mode

$$
\begin{aligned}
\dot{a}= & -\frac{\kappa_{\mathrm{a}}}{2} a-i G_{+}\left(b^{\dagger}+b \exp \left[-2 i \omega_{\mathrm{m}} t\right]\right) \\
& +\sqrt{\kappa_{\mathrm{e}, \mathrm{a}}} a_{\mathrm{i}}+\sqrt{\kappa_{\mathrm{i}, \mathrm{a}}} a_{\mathrm{I}}, \\
\dot{c}= & -\frac{\kappa_{\mathrm{c}}}{2} c-i G_{-}\left(b^{\dagger} \exp \left[2 i \omega_{\mathrm{m}} t\right]+b\right) \\
& +\sqrt{\kappa_{\mathrm{e}, \mathrm{c}}} c_{\mathrm{i}}+\sqrt{\kappa_{\mathrm{i}, \mathrm{c}}} c_{\mathrm{I}}, \\
\dot{b}= & -\frac{\gamma}{2} b-i G_{+}\left(a^{\dagger}+a \exp \left[-2 i \omega_{\mathrm{m}} t\right]\right) \\
& -i G_{-}\left(c+c^{\dagger} \exp \left[2 i \omega_{\mathrm{m}} t\right]\right)+\sqrt{\gamma} b_{\mathrm{i}},
\end{aligned}
$$

the RWA approximation consists of neglecting the (fastrotating) time-dependent terms in Eqs. (5a)-(5c), leading to 
the following simplified EOMs:

$$
\begin{aligned}
& \dot{a}=-\frac{\kappa_{\mathrm{a}}}{2} a-i G_{+} b^{\dagger}+\sqrt{\kappa_{\mathrm{e}, \mathrm{a}}} a_{\mathrm{i}}+\sqrt{\kappa_{\mathrm{i}, \mathrm{a}}} a_{\mathrm{I}}, \\
& \dot{c}=-\frac{\kappa_{\mathrm{c}}}{2} c-i G_{-} b+\sqrt{\kappa_{\mathrm{e}, \mathrm{c}}} c_{\mathrm{i}}+\sqrt{\kappa_{\mathrm{i}, \mathrm{c}}} c_{\mathrm{I}}, \\
& \dot{b}=-\frac{\gamma}{2} b-i G_{+} a^{\dagger}-i G_{-} c+\sqrt{\gamma} b_{\mathrm{i}} .
\end{aligned}
$$

We rewrite Eqs. (6a)-(6c) in terms of two Bogoliubov operators

$$
\begin{aligned}
& \eta_{\mathrm{a}}=\cosh \xi c+\sinh \xi a^{\dagger}, \\
& \eta_{\mathrm{c}}=\cosh \xi a+\sinh \xi c^{\dagger},
\end{aligned}
$$

where $\cosh \xi=G_{-} / \mathcal{G}, \quad \sinh \xi=G_{+} / \mathcal{G}, \quad$ with $\quad \mathcal{G}=$ $\left(G_{-}^{2}-G_{+}^{2}\right)^{1 / 2}$ and rewrite Eqs. (6a)-(6c) in terms of the Bogoliubov modes $\eta_{\mathrm{a}}$ and $\eta_{\mathrm{c}}$ as

$$
\begin{aligned}
\dot{\eta}_{\mathrm{a}} & =-\frac{\kappa}{2} \eta_{\mathrm{a}}-i \mathcal{G} b+\sqrt{\kappa_{\mathrm{e}}} \eta_{\mathrm{a}, \mathrm{i}}+\sqrt{\kappa_{\mathrm{i}}} \eta_{\mathrm{a}, \mathrm{I}}, \\
\dot{\eta}_{\mathrm{c}} & =-\frac{\kappa}{2} \eta_{\mathrm{c}}+\sqrt{\kappa_{\mathrm{e}}} \eta_{\mathrm{c}, \mathrm{i}}+\sqrt{\kappa_{\mathrm{i}}} \eta_{\mathrm{c}, \mathrm{I}}, \\
\dot{b} & =-\frac{\gamma}{2} b-i \mathcal{G} \eta_{\mathrm{a}}+\sqrt{\gamma} b_{\mathrm{i}},
\end{aligned}
$$

where $\eta_{\mathrm{a}, \mathrm{i}}=\cosh \xi c_{\mathrm{i}}+\sinh \xi a_{\mathrm{i}}^{\dagger}, \eta_{\mathrm{c}, \mathrm{i}}=\cosh \xi a_{\mathrm{i}}+\sinh \xi c_{\mathrm{i}}^{\dagger}$. Equations (8a)-(8c) thus show that it is possible to recast the problem in terms of the dynamics of two operators $\left(\eta_{\mathrm{a}}\right.$ and $\left.\eta_{\mathrm{c}}\right)$ resulting from the action of a two-mode squeezing operator on the original field operators, suggesting that the output modes of the field are entangled and therefore that, potentially, nonlocal correlations are present. For an incoming signal at the resonance frequency of either cavity, the RWA analysis of the problem allows us to establish that, in the limit of large cooperativity $\left(C_{-}=4 G_{-}^{2} / \kappa \gamma \gg 1\right)$, we have that $A_{\mathrm{d}}=$ $2 r_{\mathrm{e}} /\left(1-r^{2}\right)-1, C_{\mathrm{d}}=-2 r_{\mathrm{e}} r^{2} /\left(1-r^{2}\right)-1, A_{\mathrm{x}}=-C_{\mathrm{x}}=$ $2 r_{\mathrm{e}} r /\left(1-r^{2}\right)$, where $r=G_{+} / G_{-}$and $r_{\mathrm{e}}=\kappa_{\mathrm{e}} / \kappa$ is the ratio of the external coupling rate to the total losses of the cavities.

Nevertheless, in our analysis, unless explicitly stated, we show the results for the full solution of Eqs. (3a)-(3c) (i.e., without resorting to the RWA) and we assume that both cavities have the same environment coupling properties.

In our discussion, we consider that, in addition to the strong coherent tone $\alpha_{\mathrm{A}}$ and $\alpha_{\mathrm{C}}$, cavity $\mathrm{A}$ and cavity $\mathrm{C}$ are also driven by small coherent input fields $\alpha_{\mathrm{i}}$ and $\chi_{\mathrm{i}}$, respectively. In this scenario, the relation between input and output fields given by Eq. (4a) allows us to evaluate the response at the output of each cavity to the fields $\alpha_{\mathrm{i}}$ and $\chi_{\mathrm{i}}$. The correlations between $a_{\mathrm{o}}$ and $c_{\mathrm{o}}$ introduced by the combined dynamics of the two cavities and of the mechanical resonator represent the key ingredient for the generation of the correlations required to violate the CHSH BI.

As anticipated, the protocol that we have in mind is based on the measurement of the field intensity at two pairs of detectors D1, E1 and D2, E2 corresponding to the photodetection scheme of the Ref. [36] after mixing the signals $a_{\mathrm{o}}$ and $c_{\mathrm{o}}$ emerging from the optomechanical device with two local oscillators (LOs). This detection scheme is closely related to a balanced homodyne detection setup; in the case discussed here, however, both signals originating from the beam splitters

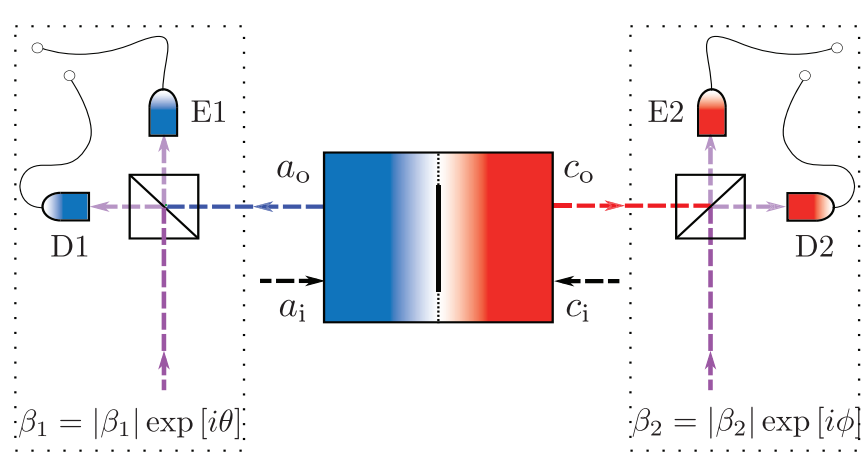

FIG. 1. Schematic of the detection scheme. Outputs of the cavities are directed to different beam splitters, where they are mixed with local oscillator (LO) fields. The mixed signals are sent to photodetectors D1, E1, D2, and E2, characterized by fields $d_{1}, e_{1}, d_{2}$, $e_{2}$, respectively. Unlike the case of (balanced) homodyne detection schemes, where the signals emerging from the two branches of each beam splitter-in our case directed towards detectors (i) D1 and E1 and (ii) D2 and E2 - are combined, we keep track of all four signals and their intensity correlations described by Eqs. (9a)-(9d).

are recorded in order to measure the required correlations. More specifically, the outputs $a_{\mathrm{o}}$ and $c_{\mathrm{o}}$ of the cavities are directed to two detectors, constituted by a beam splitter and two photodetectors each (see Fig. 1). At each detector the signal field is mixed with a coherent field of a $\mathrm{LO} \beta_{1,2}$ by a 50:50 beam splitter. The signals originating from the beam splitters are then measured at the photodetectors D1, E1, D2, and E2. To evaluate the correlations needed for the verification of the violation of the CHSH inequality, we define the correlation pairs D1, E1 and D2, E2 for different phases of the LOs as

$$
\begin{aligned}
& R_{++}(\theta, \phi)=\left\langle d_{1}^{\dagger} d_{2}^{\dagger} d_{2} d_{1}\right\rangle, \\
& R_{+-}(\theta, \phi)=\left\langle d_{1}^{\dagger} e_{2}^{\dagger} e_{2} d_{1}\right\rangle, \\
& R_{-+}(\theta, \phi)=\left\langle e_{1}^{\dagger} d_{2}^{\dagger} d_{2} e_{1}\right\rangle, \\
& R_{--}(\theta, \phi)=\left\langle e_{1}^{\dagger} e_{2}^{\dagger} e_{2} e_{1}\right\rangle,
\end{aligned}
$$

where $d_{1}, e_{1}$ and $d_{2}, e_{2}$ are the fields associated with each of pair of photodetectors, and $\theta$ and $\phi$ represent the coherent field phases of each LO. In the language of quantum optics, $R_{\mathrm{ij}}(i, j= \pm)$ represent the intensity correlations among photocurrents in the four detectors, e.g., $R_{+-}$measures correlations between the photocurrent in $\mathrm{D}_{1}$ and that in $\mathrm{E}_{2}$. The setup we are discussing here is analogous to the more conventional polarization experiments [5-9,13,14,16,17]: in these experiments each channel (D1, E1 and D2, E2) is selected by adjusting the angle of a polarizer at each detection branch. The parallel with the polarization experiments is represented by the fact that, by changing the phase of the LO, we are selecting the detection channel. We are thus essentially performing a quadrature measurement of the output fields originating from of the optomechanical system, since it is possible to relate $R_{\mathrm{ij}}$ in Eqs. (9a)-(9d) to the quadratures $X_{a}(\theta)=\left(a_{0}^{\dagger} e^{i \theta}+\right.$ $\left.a_{\mathrm{o}} e^{-i \theta}\right) / \sqrt{2}$ and $X_{c}(\phi)=\left(c_{\mathrm{o}}^{\dagger} e^{i \phi}+c_{\mathrm{o}} e^{-i \phi}\right) / \sqrt{2}$ of the output fields given in Eqs. (4a) and (4b). More specifically,focusing, for instance, on the left-hand detector in Fig. 1-we 
can write the fields $d_{1}$ and $e_{1}$ as the result of the mixing between the LO field $b_{\mathrm{LO} 1}$ and $a_{\mathrm{o}}$, the output field of cavity A, as

$$
\begin{aligned}
& d_{1}=\sqrt{\eta_{1}} a_{\mathrm{o}}+i \sqrt{1-\eta_{1}} b_{\mathrm{LO} 1}, \\
& e_{1}=\sqrt{\eta_{1}} b_{\mathrm{LO} 1}+i \sqrt{1-\eta_{1}} a_{\mathrm{o}},
\end{aligned}
$$

where $\eta_{1}$ is the transmissivity of the beam splitter associated with the left-hand detector of Fig. 1. Therefore, as discussed more in detail in Appendix $\mathrm{C}$, we can express the correlators in Eqs. (9a)-(9d) in terms of $a_{\mathrm{o}}$ and $c_{\mathrm{o}}$.

Regardless of the physical implementation, either in the optical or the microwave frequency range, the original formulation of the CHSH inequality is given by the following relation:

$$
|S|=\left|E\left(\theta_{1}, \phi_{1}\right)+E\left(\theta_{2}, \phi_{2}\right)+E\left(\theta_{1}, \phi_{2}\right)-E\left(\theta_{2}, \phi_{1}\right)\right| \leqslant 2,
$$

where, in our case, we have

$$
E(\theta, \phi)=\frac{R_{++}+R_{--}-R_{-+}-R_{+-}}{R_{++}+R_{--}+R_{-+}+R_{+-}} .
$$

In terms of correlations of the original optomechanical fields $a_{\mathrm{o}}$ and $c_{\mathrm{o}}$, Eq. (12) can be written as

$$
E=C \cos [\bar{\theta}-\bar{\phi}]+D \cos [\bar{\theta}+\bar{\phi}],
$$

where

$$
\begin{aligned}
& C=2\left|\left\langle a_{\mathrm{o}}^{\dagger} c_{\mathrm{o}}\right\rangle\right| / Z, \\
& D=2\left|\left\langle a_{\mathrm{o}} c_{\mathrm{o}}\right\rangle\right| / Z,
\end{aligned}
$$

with $Z=2\left\langle a_{\mathrm{o}}^{\dagger} c_{\mathrm{o}}^{\dagger} c_{\mathrm{o}} a_{\mathrm{o}}\right\rangle^{1 / 2}+\left\langle a_{\mathrm{o}}^{\dagger} a_{\mathrm{o}}+c_{\mathrm{o}}^{\dagger} c_{\mathrm{o}}\right\rangle$ and we have absorbed the phases of $\left\langle a_{0} c_{0}\right\rangle$ and $\left\langle a_{0}^{\dagger} c_{0}\right\rangle$ into the definitions of $\bar{\theta}$ and $\bar{\phi}$, and $\left|\beta_{1}\right|=\left|\beta_{2}\right|=|\beta|=\left\langle a_{0}^{\dagger} c_{0}^{\dagger} c_{0} a_{0}\right\rangle^{1 / 4}$. It can be shown that the latter condition maximizes the violation of the inequality given in Eq. (11)—see Appendix C.

The maxima of $S$ occur when $\bar{\theta}=0, \bar{\phi}=-\zeta, \bar{\theta}^{\prime}=-\pi / 2$, and $\bar{\phi}^{\prime}=\zeta$ and with a maximum value is given by

$$
S=2 \sqrt{2} \sqrt{C^{2}+D^{2}} \sin \left(\zeta-\zeta_{0}\right)
$$

where $\tan \left(\zeta_{0}\right)=(C+D) /(C-D)$. It is clear that the CHSH inequality given in Eq. (11), can be translated into the condition [36]

$$
\mathcal{F}=C^{2}+D^{2}<\frac{1}{2}
$$

The BI test in the optomechanical setting described by Eq. (15) can be straightforwardly evaluated by considering the definitions of $C$ and $D$, and the input-output relations given by Eqs. (2a)-(2c).

\section{RESULTS AND DISCUSSION}

In Fig. 2 we plot the value of $\mathcal{F}$ as a function of the ratio between the linearized pump strengths $r=G_{+} / G_{-}$and the coherent inputs $\alpha_{\mathrm{i}}$ and $\chi_{\mathrm{i}}$ in the absence of noise sources for parameters compatible with present-day experimental capabilities. From this figure one can see that there is a finite parameter region for which the inequality is violated. In the limit of large cooperativity $\left(C_{-} \gg 1\right)$, the maximum value of (a)

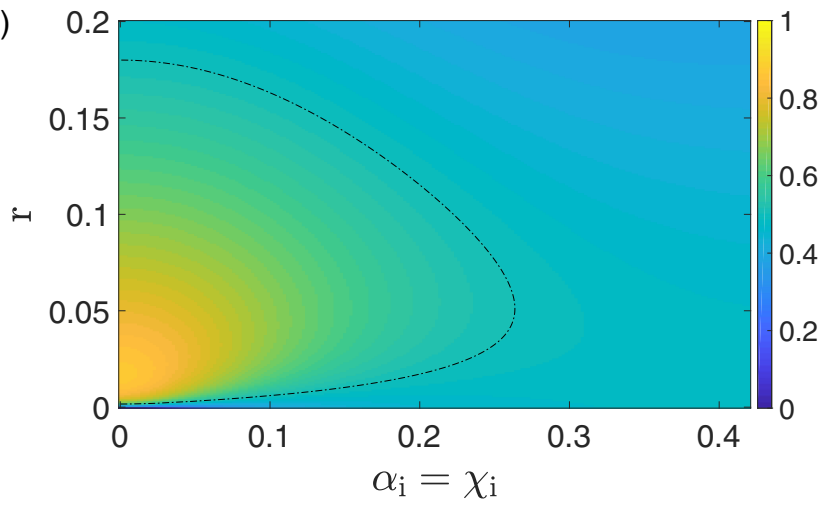

(b)

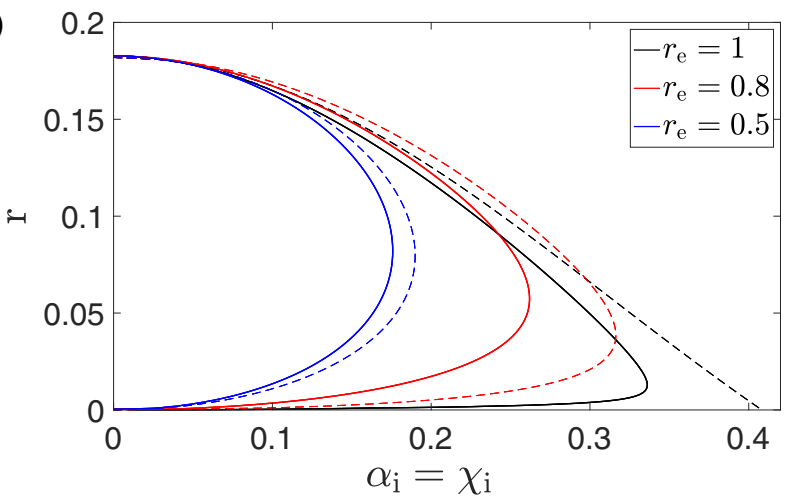

FIG. 2. (a) Value of $\mathcal{F}$ as a function of $\alpha_{\mathrm{i}}$ and $r=G_{+} / G_{-}$. Parameters: $\kappa_{\mathrm{a}}=\kappa_{\mathrm{c}}=\kappa=0.1, \kappa_{\mathrm{e}}=0.9 \kappa, \gamma=1 \times 10^{-5}$-all energies are expressed in units of $\omega_{\mathrm{m}}(\hbar=1)$ throughout the manuscript. The dashed curve corresponds to the exact boundary region $\mathcal{F}=1 / 2$, as determined from the solution of Eqs. (2a)-(2c) (b) Boundary $\mathcal{F}=$ $1 / 2$ for different values of $r_{\mathrm{e}}=\kappa_{e} / \kappa$. Smaller regions are associated with smaller values of $r_{\mathrm{e}}$. For $\alpha_{\mathrm{i}} \simeq 0.2$ it is possible to observe a crossing between boundary regions for different values of $r_{\mathrm{e}}$, hinting at a nontrivial relation between entanglement and violation of the CHSH BI (see text). The solid line corresponds to the exact boundary as in panel (a), and the dashed line corresponds to the expression given in Eq. (16).

$r$ leading to a violation of the $\mathrm{BI}$ is obtained for $\alpha_{\mathrm{i}}, \chi_{\mathrm{i}} \rightarrow 0$ and is given by $\bar{r}=(15+4 \sqrt{14})^{-1 / 2}$. Furthermore, the maximum violation of the BI $\mathcal{F}=1$ is attained for $\alpha_{\mathrm{i}}, \chi_{\mathrm{i}} \rightarrow 0$ and $r \rightarrow 0^{+}$. More specifically, for large cooperativity $\left(C_{-} \gg 1\right)$, the value of $\mathcal{F}$ exhibits a discontinuity at $\alpha_{\text {in }}\left(=\chi_{\text {in }}\right)=0$, $r=0$. As expected, for $G_{+}=0(r=0)$ modes $a_{o}$ and $c_{o}$ are not entangled and $\mathcal{F}=0$.

We note here that the $r$ dependence of the function $\mathcal{F}$ is contrasted by the $r$ dependence of entanglement. From the definition of the parameters $A_{\mathrm{d}}$ and $A_{\mathrm{x}}$, following Eq. (4a), it is possible to see that, since the squeezing parameter $z=$ $\operatorname{arctanh}\left[A_{\mathrm{x}} / A_{\mathrm{d}}\right] \rightarrow \infty$ for $r \rightarrow 1^{-}$, one obtains an infinitely squeezed state in this regime. This seemingly contradictory conclusion, analogous to the one derived in Refs. [36,37], is, however, corroborated by observing that, for mixed states, the relation between entanglement and nonlocality exhibits aspects that are still not fully understood [3]: In particular it can be shown that maximally entangled states $\left(r \rightarrow 1^{-}\right.$, in our case) do not necessarily violate locality constraints, which, conversely, can be violated by less entangled states [43-45]. 
In our setup, this complex interplay between entanglement and nonlocality is further exemplified by the crossing between the $\mathcal{F}=1 / 2$ boundary regions for different values of $r_{\mathrm{e}}$ : as can be seen in Fig. 2 for intermediate values of the coherent drive ( $\alpha_{\mathrm{i}} \simeq 0.1-0.2$ in this case), larger values of $r_{\mathrm{e}}$ lead to a reduction of the value of $r$ for which the violation is observed.

It is clear that a violation of the CHSH inequality is possible only for small values of the input fields $\alpha_{\mathrm{i}}$ and $\chi_{\mathrm{i}}$, and for small values of $r$, implying $\left|A_{\mathrm{d}}\right|=\left|C_{\mathrm{d}}\right| \approx 1$ and $\left|A_{\mathrm{x}}\right|=\left|C_{\mathrm{x}}\right| \ll 1$. Therefore, in spite of the fact that the setup proposed here has been used for nearly-quantum-limited amplification [33], the requirements for the observation of the violation of the BI dictate that $\left\langle a_{\mathrm{o}}^{\dagger} a_{\mathrm{o}}\right\rangle \simeq\left|A_{\mathrm{d}}\right|^{2}\left\langle a_{\mathrm{i}}^{\dagger} a_{\mathrm{i}}\right\rangle \approx 0.1$ and $\left\langle c_{\mathrm{o}}^{\dagger} c_{\mathrm{o}}\right\rangle \simeq\left|C_{\mathrm{d}}\right|^{2}\left\langle c_{\mathrm{i}}^{\dagger} c_{\mathrm{i}}\right\rangle \approx 0.1$. This condition combines the concomitant requirements that the value of $\mathcal{F}$ and the output signals have to be maximized. To gain better insight into the range of physical parameters for which the BI inequality is violated, we can establish an approximate analytical expression for the maximum value of $\alpha_{\mathrm{i}}$ violating the inequality as

$$
\alpha_{\mathrm{i}}=\sqrt{r_{\mathrm{e}} \bar{r}\left(1-4 \bar{r}-6 \bar{r}^{2}-12 \bar{r}^{3}\right) /\left(\mathcal{K}_{0} \bar{r}^{2}+\mathcal{K}_{1} \bar{r}+\mathcal{K}_{2}\right)},
$$

where $\mathcal{K}_{0}=28 r_{\mathrm{e}}^{2}, \mathcal{K}_{1}=2\left(1-2 r_{\mathrm{e}}+4 r_{\mathrm{e}}^{2}\right)$, and $\mathcal{K}_{2}=2(1-$ $\left.r_{\mathrm{e}}\right)^{2}$. Equation (16) is obtained as a second-order expansion of $\mathcal{F}$ in the input field intensity $\alpha_{i}^{2}$ evaluated here for the RWA solution of the problem.

So far, the discussion has focused on the ideal situation for which the effect of noise is negligible. In the following, we address the role played by the different environmental noise sources. In particular, we take into account the presence of a thermal environment for the mechanical resonator $\left(\bar{n}_{\mathrm{m}}\right.$, "mechanical noise"), for the two resonant cavities ( $\bar{n}_{\mathrm{i}}$, "internal noise") and to the noise associated with the coupling of the two resonant cavities to the input and output ports $\left(\bar{n}_{\mathrm{e}}\right.$, "external noise"). Without loss of generality, in Eq. (17) we have assumed that the noise temperature for the two cavities is equal and that all noise sources are independent. If we consider the effect of the noise on $\mathcal{F}$ to first order, we can write

$$
\mathcal{F}=\mathcal{F}_{0}-\mathcal{F}_{\mathrm{m}} \bar{n}_{\mathrm{m}}-\mathcal{F}_{\mathrm{e}} \bar{n}_{\mathrm{e}}-\mathcal{F}_{\mathrm{i}} \bar{n}_{\mathrm{i}},
$$

where $\mathcal{F}_{0}$ is the quantity previously considered for the violation of the BI, the second term represents the contribution associated with the mechanical noise, and the third (fourth) term describes the external (internal) noise contribution due to the thermal environment associated with the cavity modes. The sensitivity of the BI violation to the noise terms is encoded in the coefficients $\mathcal{F}_{\mathrm{e}}, \mathcal{F}_{\mathrm{i}}$, and $\mathcal{F}_{\mathrm{m}}$ : the larger the coefficients, the more each noise term contributes to the reduction of the value of $\mathcal{F}$ and, therefore, to the reduction of the region for which the $\mathrm{BI}$ is violated. An approximate expression for the factors appearing in Eq. (17) can be obtained by expanding the RWA approximation for $\mathcal{F}_{0}, \mathcal{F}_{\mathrm{m}}, \mathcal{F}_{\mathrm{e}}, \mathcal{F}_{\mathrm{i}}$, to lowest order in $1 / C_{-}$:

$$
\begin{aligned}
\mathcal{F}_{0} & =(2 r-1)^{2}+4 r^{2}, \\
\mathcal{F}_{\mathrm{m}} & =-2 \frac{(2 r-1)^{2}+2 r^{2}(10 r-1)}{C_{-}},
\end{aligned}
$$
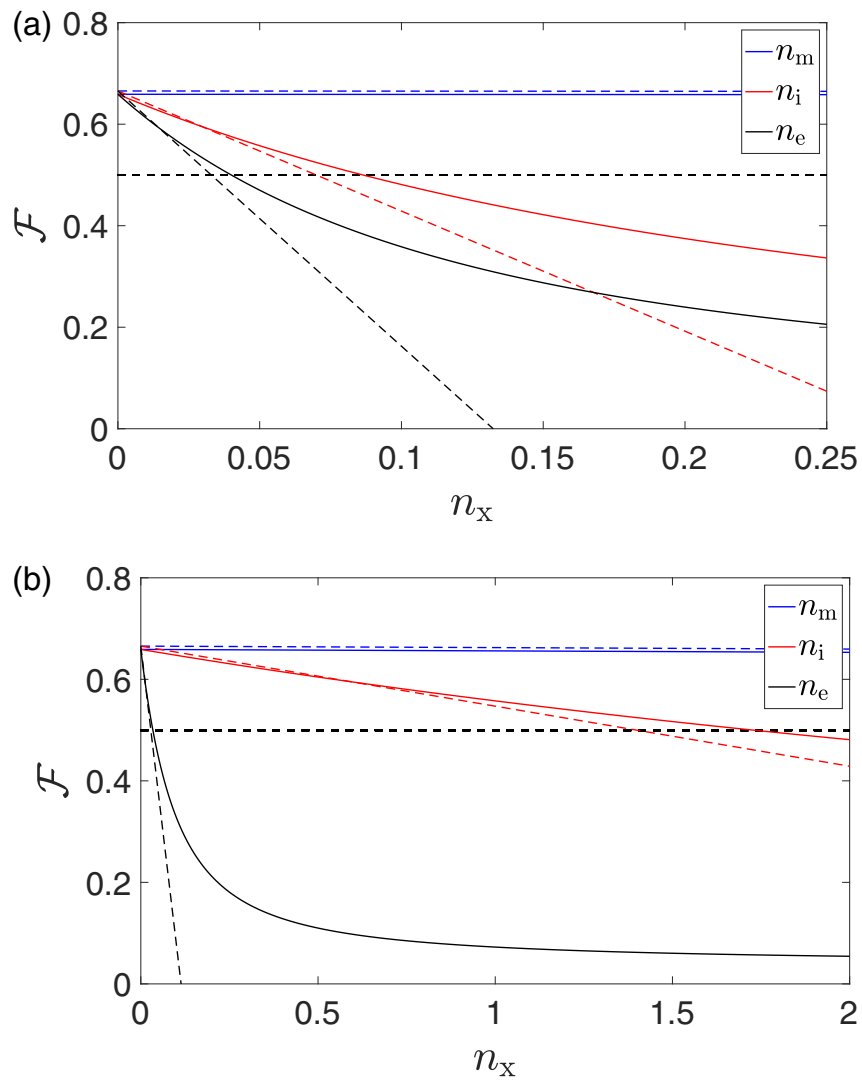

FIG. 3. Dependence of the value of $\mathcal{F}$ (for $r \rightarrow r_{\mathrm{opt}}$ and $\alpha_{\mathrm{i}}$, $\left.\chi_{i} \rightarrow 0\right)$ on the thermal population baths associated with the mechanical noise $\left(\bar{n}_{\mathrm{m}}\right.$, blue-flattest-curve), internal noise $\left(\bar{n}_{\mathrm{i}}\right.$, redintermediate-curve), and external noise $\left(\bar{n}_{\mathrm{e}}\right.$, black-steepestcurve). Solid lines correspond to the exact solution from the equations of motion with each noise source considered independently. Dashed lines are the approximations given in Eq. (7a) and Eqs. (18a)-(18d). Values of $\mathcal{F}$ above the horizontal dashed line at $\mathcal{F}=1 / 2$ correspond to the violation of the BI. Parameters: (a) $\kappa=0.01, \kappa_{e}=0.9 \kappa, G_{-}=0.2, \gamma=1 \times 10^{-5}$; (b) $\kappa_{e}=0.99 \kappa$, all other parameters are the same as in panel (a).

$$
\begin{aligned}
& \mathcal{F}_{\mathrm{e}}=-\frac{(2 r-1)^{2}+r^{2}(16 r-1)}{r} \frac{r_{\mathrm{e}}^{2}+r_{\mathrm{i}}^{2}}{r_{\mathrm{e}}}, \\
& \mathcal{F}_{\mathrm{i}}=-\frac{(2 r-1)^{2}+r^{2}(16 r-1)}{r} r_{\mathrm{i}} .
\end{aligned}
$$

The portion of the noise associated with the mechanics and described in the linear approximation by $\mathcal{F}_{\mathrm{m}}$ —see Eq. (18b) can be modified by tuning the parameter $C_{-}$. This dependence can be understood as the result of a sideband cooling process operated by the drive of cavity $\mathrm{C}$, which is driven on the red sideband. In addition, $\mathcal{F}_{\mathrm{i}}$ can be reduced by minimizing the contribution of internal losses-see Fig. 3 -, whereas $\mathcal{F}_{\mathrm{e}}$ cannot be altered significantly and thus represents the most critical parameter.

This conclusion is corroborated by Fig. 4, where we have depicted the separate effects of different noise sources on the value of $\mathcal{F}$. It is clear that the input noise $\bar{n}_{\mathrm{e}}$ represents the most sensitive parameter in the violation of the $\mathrm{CHSH}$ inequality. In this perspective, we thus select a value of $r$ that, while representing a suboptimal choice (i.e., $\mathcal{F}<1$ ) for 
(a)

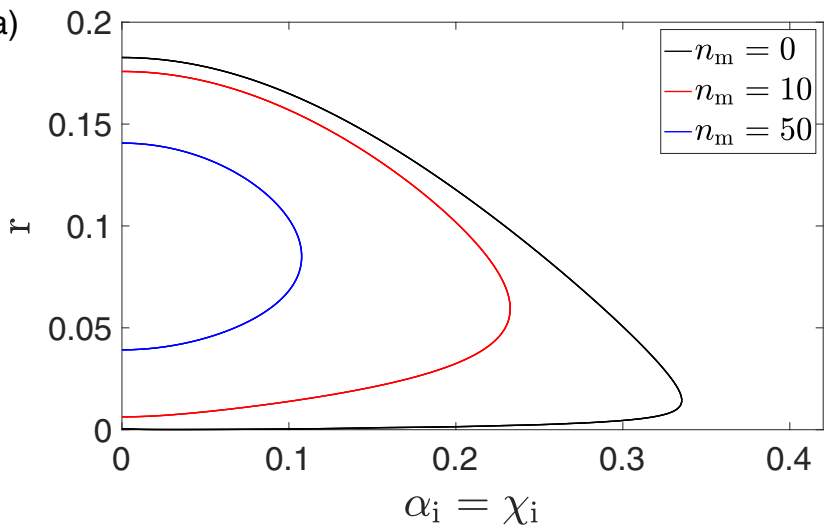

(b)

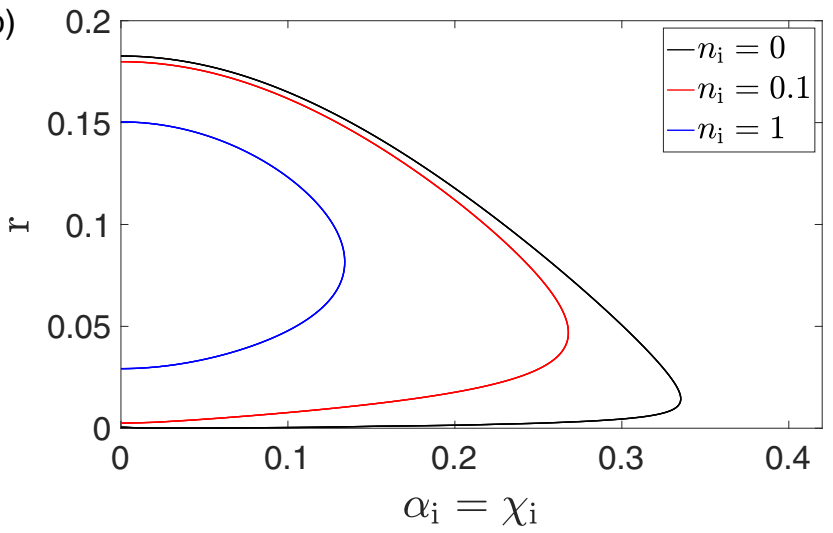

(c)

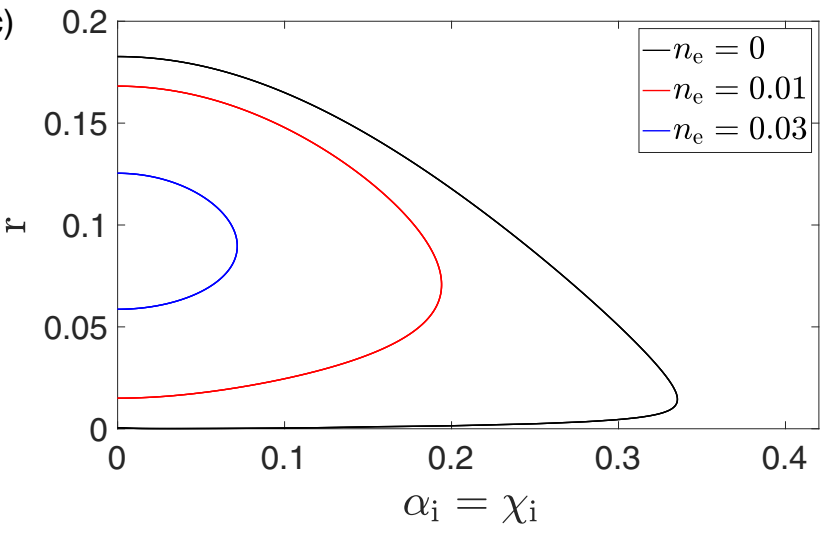

FIG. 4. Noise dependence of the $\mathcal{F}=1 / 2$ boundary in the presence of a finite coherent input. Smaller regions correspond to a large value of the noise. All parameters are the same as in Fig. 2(b).

the noiseless case, allows for the largest possible value of $n_{\mathrm{e}}$ and $n_{\mathrm{i}}$ compatible with the violation of the BI given in Eq. (15). In the linearized regime described by Eq. (17), and in the presence of cavity (external and internal) noise only, the relation describing the boundary for the violation of the BI can be expressed as

$$
\mathcal{F}_{0}(r)+\mathcal{F}_{\mathrm{e}}(r) n_{\mathrm{e}}+\mathcal{F}_{\mathrm{i}}(r) n_{\mathrm{i}}=\frac{1}{2},
$$

where we have supposed that $r_{\mathrm{e}}$ is held fixed. From Eqs. (18a)-(18d) we can write Eq. (19) as

$$
\mathcal{F}_{0}(r)-\frac{1}{2}+\mathcal{F}_{\mathrm{T}}(r) n_{\mathrm{T}}=0,
$$

where $\quad \mathcal{F}_{\mathrm{i}} / r_{\mathrm{i}}=\mathcal{F}_{\mathrm{i}} r_{\mathrm{e}} /\left(r_{\mathrm{e}}^{2}+r_{\mathrm{i}}^{2}\right)=\mathcal{F}_{\mathrm{T}} \quad$ and $\quad n_{\mathrm{T}}=\left(r_{\mathrm{e}}^{2}+\right.$ $\left.r_{\mathrm{i}}^{2}\right) / r_{\mathrm{e}} n_{\mathrm{e}}+r_{\mathrm{i}} n_{\mathrm{i}}$. From Eq. (20) $n_{\mathrm{T}}=\left[1 / 2-\mathcal{F}_{0}(r)\right] / \mathcal{F}_{T}(r)$ can be straightforwardly maximized, yielding the optimal value for $r=r_{\text {opt }}$.

We would like to stress, however, that the contribution associated with $n_{\mathrm{e}}$ assumes that the baths for the cavities are uncorrelated with each other, which represents a somewhatworst-case scenario. The potential presence of correlated noise can be considered, from the perspective of the BI violation, as a contribution to the input signals $\alpha_{\mathrm{i}}$ and $\chi_{\mathrm{i}}$.

For a microwave setting, we can assume that the cavity internal and external thermal populations are set by the base temperature of the dilution fridge $\left(T=7 \mathrm{mK}, \omega_{\mathrm{c}}=\right.$ $2 \pi \times 10 \mathrm{GHz}$ ) corresponding to $n_{\mathrm{i}}=n_{\mathrm{e}} \simeq 0.015$, whereas for an optical setting at room temperature $\left(T=300 \mathrm{~K}, \omega_{\mathrm{c}}=\right.$ $2 \pi \times 500 \mathrm{THz})$ we have $n_{\mathrm{i}}=n_{\mathrm{e}} \simeq 0.02$. While in both cases the deviation from ideality is significant, the $\mathrm{BI}$ is still clearly violated both for the microwave setting ( $\mathrm{F} \simeq 0.56$ for $r_{\mathrm{e}}=$ $0.9, \mathrm{~F} \simeq 0.58$ for $\left.r_{\mathrm{e}}=0.99\right)$ and for the optical case $(\mathcal{F} \simeq$ 0.59 for $r_{\mathrm{e}}=0.9, \mathcal{F} \simeq 0.60$ for $r_{\mathrm{e}}=0.99$ ). In Fig. 3, it is possible to note that, for parameters compatible with microwave realizations of the setup discussed in this article, the mechanical noise does not contribute to the reduction of $\mathcal{F}$. This effect is closely related to the physics of the quantum-limited amplifier discussed in Ref. [33]: in both cases the mechanics, while mediating the interaction required to generate the output fields, is concomitantly cooled by the pumping tones.

\section{CONCLUSION}

We have discussed here a potential CHSH Bell inequality test based on a quadrature phase coherence measurement in an optomechanical setting. We have shown that it is possible to violate the CHSH Bell inequality in an optomechanical setting by weakly driving a two-cavity, one-mechanics device. Furthermore, we have demonstrated that, while the thermal noise associated with cavities and mechanical degrees of freedom degrades the performances of the device proposed here, the latter is naturally suppressed by the working principle of our device. We hypothesize that our proposal could be implemented either in an optical or in a circuit QED setting.

\section{ACKNOWLEDGMENTS}

We thank Elli Selenius, Mika Sillanpää, and Caspar F. Ockeloen-Korppi for useful discussions. This work was supported by the Academy of Finland (Contract No. 275245) and the European Research Council (Grant No. 670743).

\section{APPENDIX A: EQUATIONS OF MOTION}

We derive here the equations of motion for the two cavities, one mechanical resonator system given in Eqs. (2a) and (2c) of the main text. In the presence of a strong coherent tones at blue (red) sideband for cavity A (C), the quantum Langevin equations associated with the Hamiltonian given in Eq. (1) of the main text can be written as

$$
\begin{aligned}
\dot{a}= & -\left(i \omega_{\mathrm{a}}+\frac{\kappa_{\mathrm{a}}}{2}\right) a-i g_{\mathrm{a}} a\left(b+b^{\dagger}\right) \\
& +\sqrt{\kappa_{\mathrm{e}, \mathrm{a}}} a_{\mathrm{i}}+\sqrt{\kappa_{\mathrm{i}, \mathrm{a}}} a_{\mathrm{I}},
\end{aligned}
$$




$$
\begin{aligned}
\dot{c}= & -\left(i \omega_{\mathrm{c}}+\frac{\kappa_{\mathrm{c}}}{2}\right) c-i g_{\mathrm{c}} c\left(b+b^{\dagger}\right) \\
& +\sqrt{\kappa_{\mathrm{e}, \mathrm{c}}} c_{\mathrm{i}}+\sqrt{\kappa_{\mathrm{i}, \mathrm{c}}} c_{\mathrm{I}}, \\
\dot{b}= & -\left(i \omega_{\mathrm{m}}+\frac{\gamma}{2}\right) b-i g_{\mathrm{a}} a^{\dagger} a-i g_{\mathrm{c}} c^{\dagger} c+\sqrt{\gamma} b_{\mathrm{i}},
\end{aligned}
$$

where $\kappa_{\mathrm{a}}=\kappa_{\mathrm{e}, \mathrm{a}}+\kappa_{\mathrm{i}, \mathrm{a}}$ is the total cavity decay rate, with $\kappa_{\mathrm{i}, \mathrm{a}}$ and $\kappa_{\mathrm{e}, \mathrm{a}}$ being the internal and external cavity decay rates (analogous relations hold for cavity C). The fields $a_{\mathrm{i}}, c_{\mathrm{i}}$, and $b_{\mathrm{i}}$, represent the input fields driving the cavities and the mechanical resonator, whereas $a_{\mathrm{I}}$ and $c_{\mathrm{I}}$ describe the contributions from the internal noise for cavity A and cavity $\mathrm{C}$, respectively. In the main text we consider the case of a strong drive for both cavities (with amplitudes $\alpha_{\text {in,A }}$ and $\alpha_{\text {in, C }}$ at frequencies $\omega_{\mathrm{d}, \mathrm{A}}$ and $\omega_{\mathrm{d}, \mathrm{C}}$, respectively). In this case, the quantum Langevin equations given in Eqs. (A1a)-(A1c) can be linearized around the cavity fields induced by the pump tones, leading to the following expression for the steady state for the cavity fields:

$$
\begin{aligned}
& \bar{\alpha}_{\mathrm{A}}=\frac{\alpha_{\mathrm{in}, \mathrm{A}}}{\frac{\kappa_{\mathrm{a}}}{2}+i\left[\omega_{\mathrm{a}}-g_{\mathrm{a}} \alpha_{\mathrm{A}}\left(b_{\mathrm{s}}+b_{\mathrm{s}}^{*}\right)\right]} e^{-i \omega_{\mathrm{d}, \mathrm{A}} t}=\alpha_{\mathrm{A}} e^{-i \omega_{\mathrm{d}, \mathrm{A}} t}, \\
& \bar{\alpha}_{\mathrm{C}}=\frac{\alpha_{\mathrm{in}, \mathrm{C}}}{\frac{\kappa_{\mathrm{c}}}{2}+i\left[\omega_{\mathrm{c}}-g_{\mathrm{c}} \alpha_{\mathrm{C}}\left(b_{\mathrm{s}}+b_{\mathrm{s}}^{*}\right)\right]} e^{-i \omega_{\mathrm{d}, \mathrm{C}} t}=\alpha_{\mathrm{C}} e^{-i \omega_{\mathrm{d}, \mathrm{C} t}},
\end{aligned}
$$

while the equations for the fluctuations around the steady-state values are given by

$$
\begin{aligned}
\dot{a}= & -\left(i \omega_{\mathrm{a}}+\frac{\kappa_{\mathrm{a}}}{2}\right) a-i g_{\mathrm{a}} \bar{\alpha}_{\mathrm{A}}\left(b+b^{\dagger}\right)+\sqrt{\kappa_{\mathrm{e}, \mathrm{a}}} a_{\mathrm{i}}+\sqrt{\kappa_{\mathrm{i}, \mathrm{a}}} a_{\mathrm{I}}, \\
\dot{c}= & -\left(i \omega_{\mathrm{c}}+\frac{\kappa_{\mathrm{c}}}{2}\right) c-i g_{\mathrm{c}} \bar{\alpha}_{\mathrm{C}}\left(b+b^{\dagger}\right)+\sqrt{\kappa_{\mathrm{e}, \mathrm{c}}} c_{\mathrm{i}}+\sqrt{\kappa_{\mathrm{i}, \mathrm{c}}} c_{\mathrm{I}}, \\
\dot{b}= & -\left(i \omega_{\mathrm{m}}+\frac{\gamma}{2}\right) b-i g_{\mathrm{a}} \bar{\alpha}_{\mathrm{A}}\left(a+a^{\dagger}\right) \\
& -i g_{\mathrm{c}} \bar{\alpha}_{\mathrm{C}}\left(c+c^{\dagger}\right)+\sqrt{\gamma} b_{\mathrm{i}} .
\end{aligned}
$$

Moving to a frame rotating at $\omega_{\mathrm{d}, \mathrm{a}}, \omega_{\mathrm{d}, \mathrm{c}}$, and $\omega_{\mathrm{m}}$ for cavity $\mathrm{A}$, cavity $\mathrm{C}$, and mechanics respectively, by substituting the values of $\bar{\alpha}_{\mathrm{A}}$ and $\bar{\alpha}_{\mathrm{C}}$ in Eqs. (A3a)-(A3c), the corresponding linearized quantum Langevin equations for the fluctuations around the stationary values induced by the pumps [Eqs. (2a) and $(2 \mathrm{c})$ of the main text] are

$$
\begin{aligned}
\dot{a}= & \left(-i \Delta_{\mathrm{a}}-\frac{\kappa_{\mathrm{a}}}{2}\right) a-i G_{+}\left(b^{\dagger}+b\right)+\sqrt{\kappa_{\mathrm{e}, \mathrm{a}}} a_{\mathrm{i}}+\sqrt{\kappa_{\mathrm{i}, \mathrm{a}}} a_{\mathrm{I}}, \\
\dot{c}= & \left(-i \Delta_{\mathrm{c}}-\frac{\kappa_{\mathrm{c}}}{2}\right) c-i G_{-}\left(b^{\dagger}+b\right)+\sqrt{\kappa_{\mathrm{e}, \mathrm{c}}} c_{\mathrm{i}}+\sqrt{\kappa_{\mathrm{i}, \mathrm{c}}} c_{\mathrm{I}}, \\
\dot{b}= & \left(-i \omega_{\mathrm{m}}-\frac{\gamma}{2}\right) b-i G_{+}\left(a^{\dagger}+a\right) \\
& -i G_{-}\left(c^{\dagger}+c\right)+\sqrt{\gamma} b_{\mathrm{i}},
\end{aligned}
$$

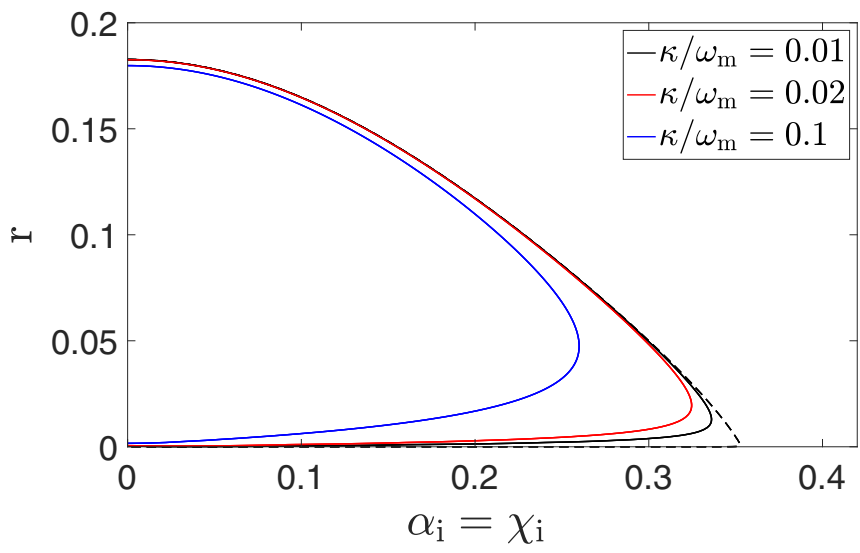

FIG. 5. Comparison between the value of $\mathcal{F}$ calculated from the full solution of the equations of motion (full lines, $\kappa=0.01,0.02$, 0.1 ; larger values correspond to smaller regions for which $\mathcal{F}>$ $1 / 2$ ), with the solution obtained in the rotating-wave approximation (dashed line).

where $G_{+}=g_{\mathrm{a}} \alpha_{\mathrm{A}}$ and $G_{-}=g_{\mathrm{c}} \alpha_{\mathrm{C}}$ are the effective linearized couplings (without loss of generality, we assume hereafter that $\left.\kappa_{\mathrm{a}}=\kappa_{\mathrm{c}}=\kappa\right)$.

\section{APPENDIX B: INPUT-OUTPUT EQUATIONS IN THE ROTATING-WAVE APPROXIMATION}

While the coefficients $A_{\mathrm{d}}, A_{\mathrm{x}}, C_{\mathrm{d}}, C_{\mathrm{x}}$-and therefore the condition expressing the violation of the BI-given in Eqs. (4a) and (4b) of the main text can be obtained without resorting to the RWA, in order to outline the essential physical process behind our proposal, we determine here the explicit analytical expression for these coefficients within the RWA.

In Fig. 5, it is possible to see how the validity of the RWA in the determination of the BI violation relies on the condition $\omega_{\mathrm{m}} \ll 1$ (good-cavity limit) as it is usually the case in the description of sideband pumping setups in optomechanics. To derive the expression of the input-output coefficients $A_{\mathrm{d}}$, $A_{\mathrm{x}}, C_{\mathrm{d}}, C_{\mathrm{x}}$ within the RWA, we define a Bogoliubov unitary transformation of the optical-mode operator as

$$
\begin{aligned}
& \eta_{\mathrm{a}}=\cosh \xi c+\sinh \xi a^{\dagger} \\
& \eta_{\mathrm{c}}=\cosh \xi a+\sinh \xi c^{\dagger}
\end{aligned}
$$

where $\cosh \xi=G_{-} / \mathcal{G}, \quad \sinh \xi=G_{+} / \mathcal{G}$ with $\mathcal{G}=$ $\left(G_{-}^{2}-G_{+}^{2}\right)^{1 / 2}$ and rewrite Eqs. (A4a)-(A4c) in terms of the Bogoliubov modes $\eta_{\mathrm{a}}$ and $\eta_{\mathrm{c}}$ as

$$
\begin{aligned}
\dot{\eta}_{\mathrm{a}} & =-\frac{\kappa}{2} \eta_{\mathrm{a}}-i \mathcal{G} b+\sqrt{\kappa_{\mathrm{e}}} \eta_{\mathrm{a}, \mathrm{i}}+\sqrt{\kappa_{\mathrm{i}}} \eta_{\mathrm{a}, \mathrm{I}} \\
\dot{\eta}_{\mathrm{c}} & =-\frac{\kappa}{2} \eta_{\mathrm{c}}+\sqrt{\kappa_{\mathrm{e}}} \eta_{\mathrm{c}, \mathrm{i}}+\sqrt{\kappa_{\mathrm{i}}} \eta_{\mathrm{c}, \mathrm{I}} \\
\dot{b} & =-\frac{\gamma}{2} b-i \mathcal{G} \eta_{\mathrm{a}}+\sqrt{\gamma} b_{\mathrm{i}}
\end{aligned}
$$

where $\eta_{\mathrm{a}, \mathrm{i}}=\cosh \xi c_{\mathrm{i}}+\sinh \xi a_{\mathrm{i}}^{\dagger}, \eta_{\mathrm{c}, \mathrm{i}}=\cosh \xi a_{\mathrm{i}}+\sinh \xi c_{\mathrm{i}}^{\dagger}$. We then transform the quantum Langevin equations of the two 
Bogoliubov modes $\eta_{\mathrm{a}}$ and $\eta_{\mathrm{c}}$ to the Fourier domain:

$$
\begin{aligned}
\eta_{\mathrm{a}}= & \frac{\chi_{\mathrm{a}}}{1+\chi_{\mathrm{m}} \chi_{\mathrm{a}} \mathcal{G}^{2}}\left(\sqrt{\kappa_{\mathrm{e}}} \eta_{\mathrm{a}, \mathrm{i}}+\sqrt{\kappa_{\mathrm{i}}} \eta_{\mathrm{a}, \mathrm{I}}\right) \\
& -i \frac{\chi_{\mathrm{m}} \chi_{a} \mathcal{G}}{1+\chi_{\mathrm{m}} \chi_{a} \mathcal{G}^{2}} \sqrt{\gamma} b_{\mathrm{i}} \\
\eta_{\mathrm{c}}= & \chi_{\mathrm{a}}\left(\sqrt{\kappa_{\mathrm{e}}} \eta_{\mathrm{c}, \mathrm{i}}+\sqrt{\kappa_{\mathrm{i}}} \eta_{\mathrm{c}, \mathrm{I}}\right),
\end{aligned}
$$

where $\chi_{\mathrm{m}}=\left(\frac{\gamma}{2}-i \omega\right)^{-1}$ and $\chi_{\mathrm{a}}=\left(\frac{\kappa}{2}-i \omega\right)^{-1}$. Since, according to the input-output theory [42], the operator for the output field is related to the cavity and to the input noise operator by the relation $a_{\mathrm{o}}=\sqrt{\kappa_{\mathrm{e}}} a-a_{\mathrm{i}}$ and $c_{\mathrm{o}}=\sqrt{\kappa_{\mathrm{e}}} c-c_{\mathrm{i}}$ by using the transformation $a=\cosh \xi \eta_{\mathrm{c}}-\sinh \xi \eta_{\mathrm{a}}^{\dagger}$ and $c=$ $\cosh \xi \eta_{\mathrm{a}}-\sinh \xi \eta_{\mathrm{c}}^{\dagger}$, the outputs of the two cavity modes can be written as

$$
\begin{aligned}
& a_{\mathrm{o}}=\left(\kappa_{\mathrm{e}} \mathcal{A}_{\mathrm{aa}}-1\right) a_{\mathrm{i}}+\kappa_{\mathrm{e}} \mathcal{A}_{\mathrm{ac}} c_{\mathrm{i}}^{\dagger}+\sqrt{\kappa_{\mathrm{i}} \kappa_{\mathrm{e}}} \mathcal{A}_{\mathrm{aa}} a_{\mathrm{I}}+\sqrt{\kappa_{\mathrm{i}} \kappa_{\mathrm{e}}} \mathcal{A}_{\mathrm{ac}} c_{\mathrm{I}}^{\dagger}+i \sqrt{\gamma \kappa_{\mathrm{e}}} \frac{G_{+}}{\left(\chi_{\mathrm{a}} \chi_{\mathrm{m}}\right)^{-1}+\mathcal{G}^{2}} b_{\mathrm{i}}^{\dagger}, \\
& c_{\mathrm{o}}=\left(\kappa_{\mathrm{e}} \mathcal{A}_{\mathrm{cc}}-1\right) c_{\mathrm{i}}+\kappa_{\mathrm{e}} \mathcal{A}_{\mathrm{ca}} a_{\mathrm{i}}^{\dagger}+\sqrt{\kappa_{\mathrm{i}} \kappa_{\mathrm{e}}} \mathcal{A}_{\mathrm{cc}} c_{\mathrm{I}}+\sqrt{\kappa_{\mathrm{i}} \kappa_{\mathrm{e}}} \mathcal{A}_{\mathrm{ca}} a_{\mathrm{I}}^{\dagger}-i \sqrt{\gamma \kappa_{\mathrm{e}}} \frac{G_{-}}{\left(\chi_{\mathrm{a}} \chi_{\mathrm{m}}\right)^{-1}+\mathcal{G}^{2}} b_{\mathrm{i}},
\end{aligned}
$$

where

$$
\begin{array}{ll}
\mathcal{A}_{\mathrm{aa}}=\chi_{\mathrm{a}} \cosh ^{2} \xi-\chi_{\mathrm{a}}^{\mathrm{e}} \sinh ^{2} \xi, & \mathcal{A}_{\mathrm{cc}}=\chi_{\mathrm{a}}^{e} \cosh ^{2} \xi-\chi_{\mathrm{a}} \sinh ^{2} \xi, \\
\mathcal{A}_{\mathrm{ac}}=\left(\chi_{\mathrm{a}}-\chi_{\mathrm{a}}^{e}\right) \cosh \xi \sinh \xi, & \mathcal{A}_{\mathrm{ca}}=\left(\chi_{\mathrm{a}}^{\mathrm{e}}-\chi_{\mathrm{a}}\right) \cosh \xi \sinh \xi,
\end{array}
$$

and $\chi_{\mathrm{a}}^{\mathrm{e}}=\chi_{\mathrm{a}}\left(1+\mathcal{G}^{2} \chi_{\mathrm{a}} \chi_{\mathrm{m}}\right)^{-1}$ represents the effective cavity response in the presence of the two-tone optomechanical drive. It is possible to write Eq. (B4) and (B5) in a more compact form as given in Eqs. (4a) and (4b) of the main text as

$$
\begin{aligned}
& a_{\mathrm{o}}=A_{\mathrm{d}} a_{\mathrm{i}}+A_{\mathrm{x}} c_{\mathrm{i}}^{\dagger}+\mathcal{N}_{\mathrm{a}}, \\
& c_{\mathrm{o}}=C_{\mathrm{d}} c_{\mathrm{i}}+C_{\mathrm{x}} a_{\mathrm{i}}^{\dagger}+\mathcal{N}_{\mathrm{c}},
\end{aligned}
$$

where

$$
\mathcal{N}_{\mathrm{a}}=A_{\mathrm{d}, \mathrm{I}} a_{\mathrm{I}}+A_{\mathrm{x}, \mathrm{I}} c_{\mathrm{I}}^{\dagger}+A_{\mathrm{m}} b_{\mathrm{i}}^{\dagger}, \quad \mathcal{N}_{\mathrm{c}}=C_{\mathrm{d}, \mathrm{I}} c_{\mathrm{I}}+C_{\mathrm{x}, \mathrm{I}} a_{\mathrm{I}}^{\dagger}+C_{\mathrm{m}} b_{\mathrm{i}}
$$

represent the operators associated with the mechanical and cavity internal noise. Furthermore, the coefficients relating input and noise operators to the output are given by

$$
\begin{aligned}
A_{\mathrm{d}} & =\kappa_{\mathrm{e}} \mathcal{A}_{\mathrm{aa}}-1, \quad C_{\mathrm{d}}=\kappa_{\mathrm{e}} \mathcal{A}_{\mathrm{cc}}-1, \quad A_{\mathrm{m}}=+i \sqrt{\gamma \kappa_{\mathrm{e}}} G_{+} \chi_{\mathrm{a}}^{\mathrm{e}} / \chi_{\mathrm{a}}, \\
A_{\mathrm{x}} & =\kappa_{\mathrm{e}} \mathcal{A}_{\mathrm{ac}}, \quad C_{\mathrm{x}}=\kappa_{\mathrm{e}} \mathcal{A}_{\mathrm{ca}}, \quad C_{\mathrm{m}}=-i \sqrt{\gamma \kappa_{\mathrm{e}}} G_{-} \chi_{\mathrm{a}}^{\mathrm{e}} / \chi_{\mathrm{a}}, \\
A_{\mathrm{d}, \mathrm{I}} & =\sqrt{\kappa_{\mathrm{i}} \kappa_{\mathrm{e}}} \mathcal{A}_{\mathrm{aa}}, \quad C_{\mathrm{d}, \mathrm{I}}=\sqrt{\kappa_{\mathrm{i}} \kappa_{\mathrm{e}}} \mathcal{A}_{\mathrm{cc}}, \\
A_{\mathrm{x}, \mathrm{I}} & =\sqrt{\kappa_{\mathrm{i}} \kappa_{\mathrm{e}}} \mathcal{A}_{\mathrm{ac}}, \quad C_{\mathrm{x}, \mathrm{I}}=\sqrt{\kappa_{\mathrm{i}} \kappa_{\mathrm{e}}} \mathcal{A}_{\mathrm{ca}} .
\end{aligned}
$$

In the limit of large cooperativity $C_{-}=4 G^{2} / \kappa \gamma \gg 1$ and at the cavity resonance, the coefficients can be written as

$$
\begin{aligned}
A_{\mathrm{d}} & =\frac{2 r_{e}}{1-r^{2}}-1, \quad C_{\mathrm{d}}=-\frac{2 r_{e} r^{2}}{1-r^{2}}-1, \quad A_{\mathrm{x}}=\frac{2 r r_{e}}{1-r^{2}}=-C_{\mathrm{x}}, \quad A_{\mathrm{m}}=-i \frac{2 r \sqrt{r_{e}}}{\sqrt{C_{-}}\left(1-r^{2}\right)}=r C_{\mathrm{m}}, \\
A_{\mathrm{d}, \mathrm{I}} & =\frac{2 \sqrt{r_{e} r_{i}}}{1-r^{2}}, \quad C_{\mathrm{d}, \mathrm{I}}=-r^{2} A_{\mathrm{d}, \mathrm{I}}, \quad A_{\mathrm{x}, \mathrm{I}}=\frac{2 r \sqrt{r_{e} r_{i}}}{1-r^{2}}=-C_{\mathrm{x}, \mathrm{I}}
\end{aligned}
$$

where $r=G_{+} / G_{-}, r_{\mathrm{e}}=\kappa_{\mathrm{e}} / \kappa$ and $r_{\mathrm{i}}=\kappa_{\mathrm{i}} / \kappa$.

\section{APPENDIX C: CLAUSER-HORNE-SHIMONY-HOLT VIOLATION}

We derive here the relation between the usual condition for the violation of CHSH inequality expressed by Eqs. (11) and (15) of the main text. To this end, we evaluate the quantity defined in Eqs. (9a)-(9d) of the main text in terms of the output correlators of the optomechanical system. For beam splitters of transmissivity given by $\eta_{1}$ and $\eta_{2}$, the detected fields are given by

$$
\begin{aligned}
& d_{1}=\sqrt{\eta_{1}} a_{\mathrm{o}}+i \sqrt{1-\eta_{1}} b_{\mathrm{LO} 1}, \\
& d_{2}=\sqrt{\eta_{2}} c_{\mathrm{o}}+i \sqrt{1-\eta_{2}} b_{\mathrm{LO} 2}, \\
& e_{1}=\sqrt{\eta_{1}} b_{\mathrm{LO} 1}+i \sqrt{1-\eta_{1}} a_{\mathrm{o}}, \\
& e_{2}=\sqrt{\eta_{2}} b_{\mathrm{LO} 2}+i \sqrt{1-\eta_{2}} c_{\mathrm{o}},
\end{aligned}
$$


where $b_{1}$ and $b_{2}$ are the fields of the local oscillators. With the definitions given by Eqs. (C1a)-(C1d) and assuming that the LO state is described by a coherent state $\left\langle b_{\mathrm{LO} 1}\right\rangle=\beta_{1} \exp [i \theta]$, we can calculate

$$
\begin{aligned}
\left\langle d_{1}^{\dagger} d_{1}\right\rangle & =\left(1-\eta_{1}\right)\left\langle b_{\mathrm{LO} 1}^{\dagger} b_{\mathrm{LO} 1}\right\rangle+\eta_{1}\left\langle a_{\mathrm{o}}^{\dagger} a_{\mathrm{o}}\right\rangle-i \sqrt{\eta_{1}\left(1-\eta_{1}\right)}\left[\left\langle b_{\mathrm{LO} 1}^{\dagger} a_{\mathrm{o}}\right\rangle-\left\langle a_{\mathrm{o}}^{\dagger} b_{\mathrm{LO} 1}\right\rangle\right] \\
& =\left(1-\eta_{1}\right)\left|\beta_{1}\right|^{2}+\eta_{1}\left\langle a_{\mathrm{o}}^{\dagger} a_{\mathrm{o}}\right\rangle+\sqrt{\eta_{1}\left(1-\eta_{1}\right)}\left|\beta_{1}\right|\left\langle X_{a}^{\theta}\right\rangle,
\end{aligned}
$$

where

$$
X_{\mathrm{a}}^{\theta}=X^{\mathrm{a}}(\theta+\pi / 2)=-i\left(a_{0} \exp [-i \theta]-a_{\mathrm{o}}^{\dagger} \exp [i \theta]\right) .
$$

Similarly, one obtains

$$
\left\langle e_{1}^{\dagger} e_{1}\right\rangle=\eta_{1}\left|\beta_{1}\right|^{2}+\left(1-\eta_{1}\right)\left\langle a_{\mathrm{o}}^{\dagger} a_{\mathrm{o}}\right\rangle-\sqrt{\eta_{1}\left(1-\eta_{1}\right)}\left|\beta_{1}\right|\left\langle X_{\mathrm{a}}^{\theta}\right\rangle,
$$

and analogously for detector 2 .

In addition to the intensities at the detectors D1, D2, E1, E2 we have to evaluate the correlations among them. To this end we evaluate the full expression for $\left\langle d_{1}^{\dagger} d_{2}^{\dagger} d_{2} d_{1}\right\rangle$, which is given by

$$
\begin{aligned}
R_{++}(\theta, \phi)= & \left\langle d_{1}^{\dagger} d_{2}^{\dagger} d_{2} d_{1}\right\rangle=\left(1-\eta_{1}\right)\left(1-\eta_{2}\right)\left\langle b_{\mathrm{LO} 1}^{\dagger} b_{\mathrm{LO} 2}^{\dagger} b_{\mathrm{LO} 2} b_{\mathrm{LO} 1}\right\rangle \\
& +i \sqrt{\eta_{1}\left(1-\eta_{1}\right)}\left(1-\eta_{2}\right)\left(\left\langle a_{\mathrm{o}}^{\dagger} b_{\mathrm{LO} 2}^{\dagger} b_{\mathrm{LO} 2} b_{\mathrm{LO} 1}\right\rangle-\left\langle b_{\mathrm{LO} 1}^{\dagger} b_{\mathrm{LO} 2}^{\dagger} b_{\mathrm{LO} 2} a_{\mathrm{o}}\right\rangle\right) \\
& +i \sqrt{\eta_{2}\left(1-\eta_{2}\right)}\left(1-\eta_{1}\right)\left(\left\langle b_{\mathrm{LO} 1}^{\dagger} c_{\mathrm{o}}^{\dagger} b_{\mathrm{LO} 2} b_{\mathrm{LO} 1}\right\rangle-\left\langle b_{\mathrm{LO} 1}^{\dagger} b_{\mathrm{LO} 2}^{\dagger} c_{\mathrm{o}} b_{\mathrm{LO} 1}\right\rangle\right) \\
& +\eta_{1}\left(1-\eta_{2}\right)\left\langle a_{\mathrm{o}}^{\dagger} b_{\mathrm{LO} 2}^{\dagger} b_{\mathrm{LO} 2} a_{\mathrm{o}}\right\rangle+\eta_{2}\left(1-\eta_{1}\right)\left\langle b_{\mathrm{LO} 1}^{\dagger} c_{\mathrm{o}}^{\dagger} c_{\mathrm{o}} b_{\mathrm{LO} 1}\right\rangle \\
& -\sqrt{\eta_{1} \eta_{2}} \sqrt{\left(1-\eta_{1}\right)\left(1-\eta_{2}\right)}\left(\left\langle b_{\mathrm{LO} 1}^{\dagger} b_{\mathrm{LO} 2}^{\dagger} c_{\mathrm{o}} a_{\mathrm{o}}\right\rangle+\left\langle a_{\mathrm{o}}^{\dagger} c_{\mathrm{o}}^{\dagger} b_{\mathrm{LO} 2} b_{\mathrm{LO} 1}\right\rangle\right. \\
& \left.-\left\langle b_{\mathrm{LO} 1}^{\dagger} c_{\mathrm{o}}^{\dagger} b_{\mathrm{LO} 2} a_{\mathrm{o}}\right\rangle-\left\langle a_{\mathrm{o}}^{\dagger} b_{\mathrm{LO} 2}^{\dagger} c_{\mathrm{o}} b_{\mathrm{LO} 1}\right\rangle\right)+i \sqrt{\eta_{1}\left(1-\eta_{1}\right)} \eta_{2}\left(\left\langle a_{\mathrm{o}}^{\dagger} c_{\mathrm{o}}^{\dagger} c_{\mathrm{o}} b_{\mathrm{LO} 1}\right\rangle-\left\langle b_{\mathrm{LO} 1}^{\dagger} c_{\mathrm{o}}^{\dagger} c_{\mathrm{o}} a_{\mathrm{o}}\right\rangle\right) \\
& +i \sqrt{\eta_{2}\left(1-\eta_{2}\right)} \eta_{1}\left(\left\langle a_{\mathrm{o}}^{\dagger} c_{\mathrm{o}}^{\dagger} b_{\mathrm{LO} 2} a_{\mathrm{o}}\right\rangle-\left\langle a_{\mathrm{o}}^{\dagger} b_{\mathrm{LO} 2}^{\dagger} c_{\mathrm{o}} a_{\mathrm{o}}\right\rangle\right)+\eta_{1} \eta_{2}\left\langle a_{\mathrm{o}}^{\dagger} c_{\mathrm{o}}^{\dagger} c_{\mathrm{o}} a_{\mathrm{o}}\right\rangle
\end{aligned}
$$

and, since we assume the LO to be in a coherent state, we have that $b_{\mathrm{LO} 1} \rightarrow\left|\beta_{1}\right| \exp [i \theta], b_{\mathrm{LO} 2} \rightarrow\left|\beta_{2}\right| \exp [i \phi]$, we get

$$
\begin{aligned}
R_{++}(\theta, \phi)= & \left\langle d_{1}^{\dagger} d_{2}^{\dagger} d_{2} d_{1}\right\rangle \\
= & \left(1-\eta_{1}\right)\left(1-\eta_{2}\right)\left|\beta_{1} \beta_{2}\right|^{2} \\
& +\left(1-\eta_{2}\right) \sqrt{\eta_{1}\left(1-\eta_{1}\right)}\left|\beta_{2}\right|^{2}\left|\beta_{1}\right|\left\langle X_{\mathrm{a}}^{\theta}\right\rangle+\left(1-\eta_{1}\right) \sqrt{\eta_{2}\left(1-\eta_{2}\right)}\left|\beta_{1}\right|^{2}\left|\beta_{2}\right|\left|X_{\mathrm{c}}^{\phi}\right\rangle \\
& +\sqrt{\eta_{1} \eta_{2}} \sqrt{\left(1-\eta_{1}\right)\left(1-\eta_{2}\right)}\left|\beta_{1} \beta_{2}\right|\left\langle: X_{\mathrm{a}}^{\theta} X_{\mathrm{c}}^{\phi}:\right\rangle+\eta_{1}\left(1-\eta_{2}\right)\left|\beta_{2}\right|^{2}\left\langle a_{\mathrm{o}}^{\dagger} a_{\mathrm{o}}\right\rangle+\eta_{2}\left(1-\eta_{1}\right)\left|\beta_{1}\right|^{2}\left\langle c_{\mathrm{o}}^{\dagger} c_{\mathrm{o}}\right\rangle \\
& +\eta_{2} \sqrt{\eta_{1}\left(1-\eta_{1}\right)}\left|\beta_{1}\right|\left\langle: X_{\mathrm{a}}^{\theta} c_{\mathrm{o}}^{\dagger} c_{\mathrm{o}}:\right\rangle+\eta_{1} \sqrt{\eta_{2}\left(1-\eta_{2}\right)}\left|\beta_{2}\right|\left\langle: X_{\mathrm{c}}^{\phi} a_{\mathrm{o}}^{\dagger} a_{\mathrm{o}}:\right\rangle+\eta_{1} \eta_{2}\left\langle a_{\mathrm{o}}^{\dagger} c_{\mathrm{o}}^{\dagger} c_{\mathrm{o}} a_{\mathrm{o}}\right\rangle,
\end{aligned}
$$

where with $\langle::\rangle$ we denote normal ordering, i.e.

$$
\left\langle: X_{\mathrm{a}}^{\theta} X_{\mathrm{c}}^{\phi}:\right\rangle=-\left\langle a_{\mathrm{o}}^{\dagger} c_{\mathrm{o}}^{\dagger} \exp [i(\theta+\phi)]+c_{\mathrm{o}} a_{\mathrm{o}} \exp [-i(\theta+\phi)]-c_{\mathrm{o}}^{\dagger} a_{\mathrm{o}} \exp [-i(\theta-\phi)]-a_{\mathrm{o}}^{\dagger} c_{\mathrm{o}} \exp [i(\theta-\phi)]\right\rangle .
$$

The other terms are obtained replacing (where appropriate) $\sqrt{\eta_{1}} \rightarrow i \sqrt{1-\eta_{i}}$ and $\sqrt{1-\eta_{i}} \rightarrow-i \sqrt{\eta_{1}}$ in Eqs. (C4) and (C5). Using the expression of $R_{ \pm \pm}(\theta, \phi)$ given by Eq. (C5) and assuming $50: 50$ beam splitters, i.e., $\eta_{1}=\eta_{2}=1 / 2$, the correlation coefficient $E(\theta, \phi)$ in Eq. (12) of the main text can be written as

$$
E(\theta, \phi)=\frac{\left|\beta_{1} \beta_{2}\right|\left|: X_{\mathrm{a}}^{\theta} X_{\mathrm{c}}^{\phi}:\right\rangle}{\left|\beta_{1}\right|^{2}\left|\beta_{2}\right|^{2}+\left|\beta_{1}\right|^{2}\left\langle c_{\mathrm{o}}^{\dagger} c_{\mathrm{o}}\right\rangle+\left|\beta_{2}\right|^{2}\left\langle a_{\mathrm{o}}^{\dagger} a_{\mathrm{o}}\right\rangle+\left\langle a_{\mathrm{o}}^{\dagger} c_{\mathrm{o}}^{\dagger} c_{\mathrm{o}} a_{\mathrm{o}}\right\rangle} .
$$

In addition, it is possible to show [36] that the optimal value of the local oscillators for the violation of the Bell inequality is given by $\beta_{1}=\beta_{2}=\left\langle a_{0}^{\dagger} c_{0}^{\dagger} c_{0} a_{0}\right\rangle^{1 / 4}$. At this point, with the expression of the correlators given in Eqs. (C2)-(C6), we are in the position to express the correlation function $E(\theta, \phi)$ as

$$
E(\theta, \phi)=C \cos (\bar{\theta}-\bar{\phi})+D \cos (\bar{\theta}+\bar{\phi}),
$$

where $\bar{\theta}-\bar{\phi}=\theta-\phi-\arg \left\langle a_{0}^{\dagger} c_{\mathrm{o}}\right\rangle, \bar{\theta}+\bar{\phi}=\theta+\phi-\arg \left\langle a_{0}^{\dagger} c_{0}^{\dagger}\right\rangle$.

The maxima of $S$ occur when $\bar{\theta}=0, \bar{\phi}=-\zeta, \bar{\theta}^{\prime}=-\pi / 2$ and $\bar{\phi}^{\prime}=\zeta$ and with a maximum value given by

$$
S=2 \sqrt{2} \sqrt{C^{2}+D^{2}} \sin \left(\zeta-\zeta_{0}\right),
$$

where $\tan \left(\zeta_{0}\right)=(C+D) /(C-D)$. The CHSH inequality, as expressed in Eq. (11), can be written as

$$
\mathcal{F}=C^{2}+D^{2}<\frac{1}{2}
$$

as given in Eq. (15) of the main text. 


\section{APPENDIX D: OUTPUT FIELD CORRELATORS}

To verify the violation of the $\mathrm{CHSH}$ inequality in the setup described in the text, we evaluate

$$
\begin{aligned}
& C=\frac{2\left|\left\langle a_{\mathrm{o}}^{\dagger} c_{\mathrm{o}}\right\rangle\right|}{2 \sqrt{\left\langle a_{\mathrm{o}}^{\dagger} c_{\mathrm{o}}^{\dagger} c_{\mathrm{o}} a_{\mathrm{o}}\right\rangle}+\left\langle c_{o}^{\dagger} c_{o}\right\rangle+\left\langle a_{\mathrm{o}}^{\dagger} a_{\mathrm{o}}\right\rangle}, \\
& D=\frac{2\left|\left\langle a_{\mathrm{o}} c_{\mathrm{o}}\right\rangle\right|}{2 \sqrt{\left\langle a_{\mathrm{o}}^{\dagger} c_{\mathrm{o}}^{\dagger} c_{\mathrm{o}} a_{\mathrm{o}}\right\rangle}+\left\langle c_{o}^{\dagger} c_{o}\right\rangle+\left\langle a_{\mathrm{o}}^{\dagger} a_{\mathrm{o}}\right\rangle},
\end{aligned}
$$

in the presence of two weak coherent drives for each cavity. In addition we consider the possibility of the presence of thermal noise for the mechanics and both cavities. The latter can be divided into external, i.e., incoming through the driving ports, or internal. In this case, we can write the input fields as $a_{\mathrm{i}}=\alpha_{\mathrm{i}}+a_{\mathrm{E}}$ and $c_{\mathrm{i}}=\chi_{\mathrm{i}}+c_{\mathrm{E}}$, where $\chi \mathrm{i}$ and $\alpha_{\mathrm{i}}$ represent the weak coherent drives, while $a_{\mathrm{E}}$ and $c_{\mathrm{E}}$ are the operators associated with the external thermal noise.

In this framework, the correlations required to evaluate the $\mathrm{CHSH}$ inequality are given by

$$
\begin{aligned}
&\left\langle a_{\mathrm{o}}^{\dagger} a_{\mathrm{o}}\right\rangle=\left|A_{\mathrm{d}}\right|^{2}\left(\left|\alpha_{\mathrm{i}}\right|^{2}+\bar{n}_{\mathrm{e}, \mathrm{a}}\right)+\left|A_{\mathrm{x}}\right|^{2}\left(\left|\chi_{\mathrm{i}}\right|^{2}+\bar{n}_{\mathrm{e}, \mathrm{c}}+1\right)+A_{\mathrm{d}}^{*} A_{\mathrm{x}} \alpha_{\mathrm{i}}^{*} \chi_{\mathrm{i}}^{*}+A_{\mathrm{x}}^{*} A_{\mathrm{d}} \alpha_{\mathrm{i}} \chi_{\mathrm{i}} \\
&+\left|A_{\mathrm{d}, \mathrm{I}}\right|^{2} \bar{n}_{\mathrm{i}, \mathrm{a}}+\left|A_{\mathrm{x}, \mathrm{I}}\right|^{2}\left(\bar{n}_{\mathrm{i}, \mathrm{c}}+1\right)+\left|A_{\mathrm{m}}\right|^{2}\left(\bar{n}_{\mathrm{m}}+1\right), \\
&\left\langle c_{\mathrm{o}}^{\dagger} c_{\mathrm{o}}\right\rangle=\left|C_{\mathrm{d}}\right|^{2}\left(\left|\chi_{\mathrm{i}}\right|^{2}+\bar{n}_{\mathrm{e}, \mathrm{c}}\right)+\left|C_{\mathrm{x}}\right|^{2}\left(\left|\alpha_{\mathrm{i}}\right|^{2}+\bar{n}_{\mathrm{e}, \mathrm{a}}+1\right)+C_{\mathrm{d}}^{*} C_{\mathrm{x}} \alpha_{\mathrm{i}}^{*} \chi_{\mathrm{i}}^{*}+C_{\mathrm{x}}^{*} C_{\mathrm{d}} \alpha_{\mathrm{i}} \chi_{\mathrm{i}} \\
&+\left|C_{\mathrm{d}, \mathrm{I}}\right|^{2} \bar{n}_{\mathrm{i}, \mathrm{c}}+\left|C_{\mathrm{x}, \mathrm{I}}\right|^{2}\left(\bar{n}_{\mathrm{i}, \mathrm{a}}+1\right)+\left|C_{\mathrm{m}}\right|^{2} \bar{n}_{\mathrm{m}}, \\
&\left\langle a_{\mathrm{o}}^{\dagger} c_{\mathrm{o}}\right\rangle=A_{\mathrm{d}}^{*} C_{\mathrm{x}} \alpha_{\mathrm{i}}^{* 2}+\left(A_{\mathrm{d}}^{*} C_{\mathrm{d}}+A_{\mathrm{x}}^{*} C_{\mathrm{x}}\right) \alpha_{\mathrm{i}}^{*} \chi_{\mathrm{i}}+A_{\mathrm{x}}^{*} C_{\mathrm{d}} \chi_{\mathrm{i}}^{2}, \\
&\left\langle a_{\mathrm{o}} c_{\mathrm{o}}\right\rangle= A_{\mathrm{d}} C_{\mathrm{x}}\left(\left|\alpha_{\mathrm{i}}\right|^{2}+\bar{n}_{\mathrm{e}, \mathrm{a}}+1\right)+A_{\mathrm{x}} C_{\mathrm{d}}\left(\left|\chi_{\mathrm{i}}\right|^{2}+\bar{n}_{\mathrm{e}, \mathrm{c}}\right)+A_{\mathrm{d}} C_{\mathrm{d}} \alpha_{\mathrm{i}} \chi_{\mathrm{i}}+A_{\mathrm{x}} C_{\mathrm{x}} \alpha_{\mathrm{i}}^{*} \chi_{\mathrm{i}}^{*} \\
&+A_{\mathrm{d}, \mathrm{I}} C_{\mathrm{x}, \mathrm{I}}\left(\bar{n}_{\mathrm{i}, \mathrm{a}}+1\right)+A_{\mathrm{x}, \mathrm{I}} C_{\mathrm{d}, \mathrm{I}} \bar{n}_{\mathrm{i}, \mathrm{c}}+A_{\mathrm{m}} C_{\mathrm{m}} \bar{n}_{\mathrm{m}} .
\end{aligned}
$$

Additionally, the fourth-order correlator is

$$
\begin{aligned}
& \left\langle a_{\mathrm{o}}^{\dagger} c_{\mathrm{o}}^{\dagger} a_{\mathrm{o}} c_{\mathrm{o}}\right\rangle=\left|A_{\mathrm{d}} C_{\mathrm{x}}\right|^{2}\left(\left|\alpha_{\mathrm{i}}\right|^{4}+\left|\alpha_{\mathrm{i}}\right|^{2}+4\left|\alpha_{\mathrm{i}}\right|^{2} \bar{n}_{\mathrm{e}, \mathrm{a}}+n_{\mathrm{e}, \mathrm{a}}^{2}\right) \\
& +\left|A_{\mathrm{x}} C_{\mathrm{d}}\right|^{2}\left(\left|\chi_{\mathrm{i}}\right|^{4}+3\left|\chi_{\mathrm{i}}\right|^{2}+4\left|\chi_{\mathrm{i}}\right|^{2} \bar{n}_{\mathrm{e}, \mathrm{c}}+n_{\mathrm{e}, \mathrm{c}}^{2}+2 \bar{n}_{\mathrm{e}, \mathrm{c}}+1\right) \\
& +\left|A_{\mathrm{d}}\right|^{2}\left(\left|\alpha_{\mathrm{i}}\right|+\bar{n}_{\mathrm{e}, \mathrm{a}}\right)\left[\left|C_{\mathrm{d}}\right|^{2}\left(\left|\chi_{\mathrm{i}}\right|^{2}+\bar{n}_{\mathrm{e}, \mathrm{c}}\right)+\left|C_{\mathrm{d}, \mathrm{I}}\right|^{2} \bar{n}_{\mathrm{i}, \mathrm{c}}+\left|C_{\mathrm{x}, \mathrm{I}}\right|^{2}\left(\bar{n}_{\mathrm{i}, \mathrm{a}}+1\right)+\left|C_{\mathrm{m}}\right|^{2} \bar{n}_{\mathrm{m}}\right] \\
& +\left|A_{\mathrm{x}}\right|^{2}\left(\left|\chi_{\mathrm{i}}\right|+\bar{n}_{\mathrm{e}, \mathrm{c}}+1\right)\left[\left|C_{\mathrm{x}}\right|^{2}\left(\left|\alpha_{\mathrm{i}}\right|^{2}+\bar{n}_{\mathrm{e}, \mathrm{a}}+1\right)+\left|C_{\mathrm{d}, \mathrm{I}}\right|^{2} \bar{n}_{\mathrm{i}, \mathrm{c}}+\left|C_{\mathrm{x}, \mathrm{I}}\right|^{2}\left(\bar{n}_{\mathrm{i}, \mathrm{a}}+1\right)+\left|C_{\mathrm{m}}\right|^{2} \bar{n}_{\mathrm{m}}\right] \\
& +\left|A_{\mathrm{d}, \mathrm{I}}\right|^{2} \bar{n}_{\mathrm{i}, \mathrm{a}}\left[\left|C_{\mathrm{d}}\right|^{2}\left(\left|\chi_{\mathrm{i}}\right|^{2}+\bar{n}_{\mathrm{e}, \mathrm{c}}\right)+\left|C_{\mathrm{x}}\right|^{2}\left(\left|\alpha_{\mathrm{i}}\right|^{2}+\bar{n}_{\mathrm{e}, \mathrm{a}}+1\right)+\left|C_{\mathrm{d}, \mathrm{I}}\right|^{2} \bar{n}_{\mathrm{i}, \mathrm{c}}+\left|C_{\mathrm{m}}\right|^{2} \bar{n}_{\mathrm{m}}\right] \\
& +\left|A_{\mathrm{x}, \mathrm{I}}\right|^{2}\left(\bar{n}_{\mathrm{i}, \mathrm{c}}+1\right)\left[\left|C_{\mathrm{d}}\right|^{2}\left(\left|\chi_{\mathrm{i}}\right|^{2}+\bar{n}_{\mathrm{e}, \mathrm{c}}\right)+\left|C_{\mathrm{x}}\right|^{2}\left(\left|\alpha_{\mathrm{i}}\right|^{2}+\bar{n}_{\mathrm{e}, \mathrm{a}}+1\right)+\left|C_{\mathrm{x}, \mathrm{I}}\right|^{2}\left(\bar{n}_{\mathrm{i}, \mathrm{a}}+1\right)+\left|C_{\mathrm{m}}\right|^{2} \bar{n}_{\mathrm{m}}\right] \\
& +\left|A_{\mathrm{m}}\right|^{2}\left(\bar{n}_{\mathrm{m}}+1\right)\left[\left|C_{\mathrm{d}}\right|^{2}\left(\left|\chi_{\mathrm{i}}\right|^{2}+\bar{n}_{\mathrm{e}, \mathrm{c}}\right)+\left|C_{\mathrm{x}}\right|^{2}\left(\left|\alpha_{\mathrm{i}}\right|^{2}+\bar{n}_{\mathrm{e}, \mathrm{a}}+1\right)+\left|C_{\mathrm{d}, \mathrm{I}}\right|^{2} \bar{n}_{\mathrm{i}, \mathrm{c}}+\left|C_{\mathrm{x}, \mathrm{I}}\right|^{2}\left(\bar{n}_{\mathrm{i}, \mathrm{a}}+1\right)\right] \\
& +\left|A_{\mathrm{d}, \mathrm{I}} C_{\mathrm{x}, \mathrm{I}}\right|^{2} n_{\mathrm{a}}^{\mathrm{I} 2}+\left|A_{\mathrm{x}, \mathrm{I}} C_{\mathrm{d}, \mathrm{I}}\right|^{2}\left(n_{\mathrm{c}}^{\mathrm{I} 2}+1\right)+\left|A_{\mathrm{m}} C_{\mathrm{m}}\right|^{2}\left(\bar{n}_{\mathrm{m}}^{2}+2 \bar{n}_{\mathrm{m}}+1\right) \\
& +A_{\mathrm{d}}^{*} C_{\mathrm{d}}^{*} \alpha_{\mathrm{i}}^{*} \chi_{\mathrm{i}}^{*}\left[A_{\mathrm{x}} C_{\mathrm{x}} \alpha_{\mathrm{i}}^{*} \chi_{\mathrm{i}}^{*}+A_{\mathrm{d}, \mathrm{I}} C_{\mathrm{x}, \mathrm{I}} \bar{n}_{\mathrm{i}, \mathrm{a}}+A_{\mathrm{x}, \mathrm{I}} C_{\mathrm{d}, \mathrm{I}}\left(\bar{n}_{\mathrm{i}, \mathrm{c}}+1\right)+A_{\mathrm{m}} C_{\mathrm{m}}\left(\bar{n}_{\mathrm{m}}+1\right)\right] \\
& +A_{\mathrm{d}}^{*} C_{\mathrm{x}}^{*}\left(\left|\alpha_{\mathrm{i}}\right|^{2}+\bar{n}_{\mathrm{e}, \mathrm{a}}\right)\left[A_{\mathrm{x}} C_{\mathrm{d}}\left(\left|\chi_{\mathrm{i}}\right|^{2}+\bar{n}_{\mathrm{e}, \mathrm{c}}+1\right)+A_{\mathrm{d}, \mathrm{I}} C_{\mathrm{x}, \mathrm{I}} \bar{n}_{\mathrm{i}, \mathrm{a}}+A_{\mathrm{x}, \mathrm{I}} C_{\mathrm{d}, \mathrm{I}}\left(\bar{n}_{\mathrm{i}, \mathrm{c}}+1\right)+A_{\mathrm{m}} C_{\mathrm{m}}\left(\bar{n}_{\mathrm{m}}+1\right)\right] \\
& +A_{\mathrm{x}}^{*} C_{\mathrm{d}}^{*}\left(\left|\chi_{\mathrm{i}}\right|^{2}+\bar{n}_{\mathrm{e}, \mathrm{c}}+1\right)\left[A_{\mathrm{d}} C_{\mathrm{x}}\left(\left|\alpha_{\mathrm{i}}\right|^{2}+\bar{n}_{\mathrm{e}, \mathrm{a}}\right)+A_{\mathrm{d}, \mathrm{I}} C_{\mathrm{x}, \mathrm{I}} \bar{n}_{\mathrm{i}, \mathrm{a}}+A_{\mathrm{x}, \mathrm{I}} C_{\mathrm{d}, \mathrm{I}}\left(\bar{n}_{\mathrm{i}, \mathrm{c}}+1\right)+A_{\mathrm{m}} C_{\mathrm{m}}\left(\bar{n}_{\mathrm{m}}+1\right)\right] \\
& +A_{\mathrm{x}}^{*} C_{\mathrm{x}}^{*} \alpha_{\mathrm{i}} \chi_{\mathrm{i}}\left[A_{\mathrm{d}} C_{\mathrm{d}} \alpha_{\mathrm{i}} \chi_{\mathrm{i}}+A_{\mathrm{d}, \mathrm{I}} C_{\mathrm{x}, \mathrm{I}} \bar{n}_{\mathrm{i}, \mathrm{a}}+A_{\mathrm{x}, \mathrm{I}} C_{\mathrm{d}, \mathrm{I}}\left(\bar{n}_{\mathrm{i}, \mathrm{c}}+1\right)+A_{\mathrm{m}} C_{\mathrm{m}}\left(\bar{n}_{\mathrm{m}}+1\right)\right] \\
& +A_{\mathrm{d}, \mathrm{I}}^{*} C_{\mathrm{x}, \mathrm{I}}^{*} \bar{n}_{\mathrm{i}, \mathrm{a}}\left[A_{\mathrm{d}} C_{\mathrm{d}} \alpha_{\mathrm{i}} \chi_{\mathrm{i}}+A_{\mathrm{d}} C_{\mathrm{x}}\left(\left|\alpha_{\mathrm{i}}\right|^{2}+\bar{n}_{\mathrm{e}, \mathrm{a}}\right)+A_{\mathrm{x}} C_{\mathrm{d}}\left(\left|\chi_{\mathrm{i}}\right|^{2}+\bar{n}_{\mathrm{e}, \mathrm{c}}+1\right)+A_{\mathrm{x}} C_{\mathrm{x}} \alpha_{\mathrm{i}}^{*} \chi_{\mathrm{i}}^{*}\right. \\
& \left.+A_{\mathrm{x}, \mathrm{I}} C_{\mathrm{d}, \mathrm{I}}\left(\bar{n}_{\mathrm{i}, \mathrm{c}}+1\right)+A_{\mathrm{m}} C_{\mathrm{m}}\left(\bar{n}_{\mathrm{m}}+1\right)\right] \\
& +A_{\mathrm{x}, \mathrm{I}}^{*} C_{\mathrm{d}, \mathrm{I}}^{*}\left(\bar{n}_{\mathrm{i}, \mathrm{c}}+1\right)\left[A_{\mathrm{d}} C_{\mathrm{d}} \alpha_{\mathrm{i}} \chi_{\mathrm{i}}+A_{\mathrm{d}} C_{\mathrm{x}}\left(\left|\alpha_{\mathrm{i}}\right|^{2}+\bar{n}_{\mathrm{e}, \mathrm{a}}\right)+A_{\mathrm{x}} C_{\mathrm{d}}\left(\left|\chi_{\mathrm{i}}\right|^{2}+\bar{n}_{\mathrm{e}, \mathrm{c}}+1\right)+A_{\mathrm{x}} C_{\mathrm{x}} \alpha_{\mathrm{i}}^{*} \chi_{\mathrm{i}}^{*}\right. \\
& \left.+A_{\mathrm{d}, \mathrm{I}} C_{\mathrm{x}, \mathrm{I}} \bar{n}_{\mathrm{i}, \mathrm{a}}+A_{\mathrm{m}} C_{\mathrm{m}}\left(\bar{n}_{\mathrm{m}}+1\right)\right] \\
& +A_{\mathrm{m}}^{*} C_{\mathrm{m}}^{*}\left(\bar{n}_{\mathrm{m}}+1\right)\left[A_{\mathrm{d}} C_{\mathrm{d}} \alpha_{\mathrm{i}} \chi_{\mathrm{i}}+A_{\mathrm{d}} C_{\mathrm{x}}\left(\left|\alpha_{\mathrm{i}}\right|^{2}+\bar{n}_{\mathrm{e}, \mathrm{a}}\right)+A_{\mathrm{x}} C_{\mathrm{d}}\left(\left|\chi_{\mathrm{i}}\right|^{2}+\bar{n}_{\mathrm{e}, \mathrm{c}}+1\right)+A_{\mathrm{x}} C_{\mathrm{x}} \alpha_{\mathrm{i}}^{*} \chi_{\mathrm{i}}^{*}\right. \\
& \left.+A_{\mathrm{d}, \mathrm{I}} C_{\mathrm{x}, \mathrm{I}} \bar{n}_{\mathrm{i}, \mathrm{a}}+A_{\mathrm{x}, \mathrm{I}} C_{\mathrm{d}, \mathrm{I}}\left(\bar{n}_{\mathrm{i}, \mathrm{c}}+1\right)\right] \\
& +\left|A_{\mathrm{d}}\right|^{2} C_{\mathrm{d}}^{*} C_{\mathrm{x}} \chi_{\mathrm{i}}^{*}\left(\alpha_{\mathrm{i}}^{*}\left|\alpha_{\mathrm{i}}\right|^{2}+2 \alpha_{\mathrm{i}}^{*} \bar{n}_{\mathrm{e}, \mathrm{a}}\right)+\left|A_{\mathrm{d}}\right|^{2} C_{\mathrm{d}} C_{\mathrm{x}}^{*} \chi_{\mathrm{i}}\left(\alpha_{\mathrm{i}}\left|\alpha_{\mathrm{i}}\right|^{2}+2 \alpha_{\mathrm{i}} \bar{n}_{\mathrm{e}, \mathrm{a}}\right) \\
& +\left|A_{\mathrm{x}}\right|^{2} C_{\mathrm{d}}^{*} C_{\mathrm{x}} \alpha_{\mathrm{i}}^{*}\left(\chi_{\mathrm{i}}^{*}\left|\chi_{\mathrm{i}}\right|^{2}+2 \chi_{\mathrm{i}}^{*} \bar{n}_{\mathrm{e}, \mathrm{c}}+2 \chi_{\mathrm{i}}^{*}\right)+\left|A_{\mathrm{x}}\right|^{2} C_{\mathrm{d}} C_{\mathrm{x}}^{*} \alpha_{\mathrm{i}}\left(\chi_{\mathrm{i}}\left|\chi_{\mathrm{i}}\right|^{2}+2 \chi_{\mathrm{i}} \bar{n}_{\mathrm{e}, \mathrm{c}}+2 \chi_{\mathrm{i}}\right)
\end{aligned}
$$




$$
\begin{aligned}
& +A_{\mathrm{d}}^{*} A_{\mathrm{x}}\left|C_{\mathrm{d}}\right|^{2} \alpha_{\mathrm{i}}^{*}\left(\chi_{\mathrm{i}}^{*}\left|\chi_{\mathrm{i}}\right|^{2}+2 \chi_{\mathrm{i}}^{*} \bar{n}_{\mathrm{e}, \mathrm{c}}+\chi_{\mathrm{i}}^{*}\right)+A_{\mathrm{d}} A_{\mathrm{x}}^{*}\left|C_{\mathrm{d}}\right|^{2} \alpha_{\mathrm{i}}\left(\chi_{\mathrm{i}}\left|\chi_{\mathrm{i}}\right|^{2}+2 \chi_{\mathrm{i}} \bar{n}_{\mathrm{e}, \mathrm{c}}+\chi_{\mathrm{i}}\right) \\
& +A_{\mathrm{d}}^{*} A_{\mathrm{x}}\left|C_{\mathrm{x}}\right|^{2} \chi_{\mathrm{i}}^{*}\left(\alpha_{\mathrm{i}}^{*}\left|\alpha_{\mathrm{i}}\right|^{2}+2 \alpha_{\mathrm{i}}^{*} \bar{n}_{\mathrm{e}, \mathrm{a}}+\alpha_{\mathrm{i}}^{*}\right)+A_{\mathrm{d}} A_{\mathrm{x}}^{*}\left|C_{\mathrm{x}}\right|^{2} \chi_{\mathrm{i}}\left(\alpha_{\mathrm{i}}\left|\alpha_{\mathrm{i}}\right|^{2}+2 \alpha_{\mathrm{i}} \bar{n}_{\mathrm{e}, \mathrm{a}}+\alpha_{\mathrm{i}}\right) .
\end{aligned}
$$

[1] A. Einstein, B. Podolsky, and N. Rosen, Phys. Rev. 47, 777 (1935).

[2] J. S. Bell, Physics (Long Island City, N. Y.) 1, 195 (1964).

[3] N. Brunner et al., Rev. Mod. Phys. 86, 419 (2014).

[4] A. Acin, N. Brunner, N. Gisin, S. Massar, S. Pironio, and V. Scarani, Phys. Rev. Lett. 98, 230501 (2007).

[5] S. J. Freedman and J. F. Clauser, Phys. Rev. Lett. 28, 938 (1972).

[6] E. S. Fry and R. C. Thompson, Phys. Rev. Lett. 37, 465 (1976).

[7] A. Aspect, J. Dalibard, and G. Roger, Phys. Rev. Lett. 49, 1804 (1982).

[8] A. Aspect, P. Grangier, and G. Roger, Phys. Rev. Lett. 49, 91 (1982).

[9] G. Weihs, T. Jennewein, C. Simon, H. Weinfurter, and A. Zeilinger, Phys. Rev. Lett. 81, 5039 (1998).

[10] M. A. Rowe et al., Nature (London) 409, 791 (2001).

[11] D. N. Matsukevich, P. Maunz, D. L. Moehring, S. Olmschenk, and C. Monroe, Phys. Rev. Lett. 100, 150404 (2008).

[12] M. Ansmann et al., Nature (London) 461, 504 (2009).

[13] M. Giustina et al., Nature (London) 497, 227 (2013).

[14] B. G. Christensen et al., Phys. Rev. Lett. 111, 130406 (2013).

[15] B. Hensen et al., Nature (London) 526, 682 (2015).

[16] M. Giustina et al., Phys. Rev. Lett. 115, 250401 (2015).

[17] L. K. Shalm et al., Phys. Rev. Lett. 115, 250402 (2015).

[18] O. Thearle et al., Phys. Rev. Lett. 120, 040406 (2018).

[19] E. H. Huntington and T. C. Ralph, Phys. Rev. A 65, 012306 (2001).

[20] Z. Y. Ou and L. Mandel, Phys. Rev. Lett. 61, 50 (1988).

[21] K. Hammerer et al., in Cavity Optomechanics: Nano- and Micromechanical Resonators Interacting with Light (Springer, Berlin, Heidelberg, 2014), pp. 25-56.

[22] M. Aspelmeyer, T. J. Kippenberg, and F. Marquardt, Rev. Mod. Phys. 86, 1391 (2014).

[23] B. P. Abbott et al., Phys. Rev. Lett. 116, 061102 (2016).
[24] I. Pikovski et al., Nat. Phys. 8, 393 (2012).

[25] Y.-D. Wang and A. A. Clerk, Phys. Rev. Lett. 110, 253601 (2013).

[26] M. J. Woolley and A. A. Clerk, Phys. Rev. A 89, 063805 (2014).

[27] F. Massel, Phys. Rev. A 95, 063816 (2017).

[28] R. Riedinger et al., Nature (London) 556, 473 (2018).

[29] C. F. Ockeloen-Korppi et al., Nature (London) 556, 478 (2018).

[30] J. F. Clauser et al., Phys. Rev. Lett. 23, 880 (1969).

[31] S. Barzanjeh, M. Abdi, G. J. Milburn, P. Tombesi, and D. Vitali, Phys. Rev. Lett. 109, 130503 (2012).

[32] M. Paternostro, D. Vitali, S. Gigan, M. S. Kim, C. Brukner, J. Eisert, and M. Aspelmeyer, Phys. Rev. Lett. 99, 250401 (2007).

[33] C. F. Ockeloen-Korppi, E. Damskagg, J. M. Pirkkalainen, T. T. Heikkila, F. Massel, and M. A. Sillanpaa, Phys. Rev. X 6, 041024 (2016).

[34] V. C. Vivoli, T. Barnea, C. Galland, and N. Sangouard, Phys. Rev. Lett. 116, 070405 (2016).

[35] S. G. Hofer, K. W. Lehnert, and K. Hammerer, Phys. Rev. Lett. 116, 070406 (2016).

[36] S. M. Tan, M. J. Holland, and D. F. Walls, Opt. Commun. 77, 285 (1990).

[37] S. M. Tan, D. F. Walls, and M. J. Collett, Phys. Rev. Lett. 66, 252 (1991).

[38] C. K. Law, Phys. Rev. A 51, 2537 (1995).

[39] C. Genes et al., Adv. At. Mol. Opt. Phys. 57, 33 (2009).

[40] G. J. Milburn and M. J. Woolley, Acta Phys. Slovaca 61, 483 (2011).

[41] W. P. Bowen and G. J. Milburn, Quantum Optomechanics (CRC Press, Boca Raton, FL, 2015).

[42] D. F. Walls and G. J. Milburn, Quantum Optics (Springer, Berlin, Heidelberg, 2008).

[43] T. Vidick and S. Wehner, Phys. Rev. A 83, 052310 (2011).

[44] M. Junge and C. Palazuelos, Commun. Math. Phys. 306, 695 (2011).

[45] G. Vallone et al., Phys. Rev. A 89, 012102 (2014). 